\title{
Information-Theoretic Analysis of Underwater Acoustic OFDM Systems in Highly Dispersive Channels
}

\author{
Francois-Xavier Socheleau, ${ }^{1}$ Milica Stojanovic, ${ }^{2}$ \\ Christophe Laot, ${ }^{3}$ and Jean-Michel Passerieux ${ }^{4}$ \\ ${ }^{1}$ ENSTA Bretagne, UMR CNRS 6285 Lab-STICC, Université Européenne de Bretagne, 2 rue Francois Verny, \\ 29806 Brest Cedex 9, France \\ ${ }^{2}$ Department of Electrical and Computer Engineering, Northeastern University, Boston, MA 02115, USA \\ ${ }^{3}$ Institut Mines-Télécom, Telecom Bretagne, UMR CNRS 6285 Lab-STICC, Université Européenne de Bretagne, \\ Technopôle Brest Iroise-CS 83818, 29238 Brest Cedex, France \\ ${ }^{4}$ General Sonar Studies Group, Thales Underwater Systems, 525 route des Dolines, 06903 Sophia Antipolis Cedex, France
}

Correspondence should be addressed to Francois-Xavier Socheleau, francois-xavier.socheleau@ensta-bretagne.fr

Received 10 February 2012; Accepted 24 April 2012

Academic Editor: Konstantinos Pelekanakis

Copyright () 2012 Francois-Xavier Socheleau et al. This is an open access article distributed under the Creative Commons Attribution License, which permits unrestricted use, distribution, and reproduction in any medium, provided the original work is properly cited.

\begin{abstract}
This paper investigates the signal-to-interference ratio and the achievable rates of underwater acoustic (UA) OFDM systems over channels where time and frequency dispersion are high enough that (i) neither the transmitter nor the receiver can have a priori knowledge of the channel state information and (ii) intersymbol/intercarrier interference (ISI/ICI) cannot be neglected in the information-theoretic treatment. The goal of this study is to obtain a better understanding of the interplay between interference and the achievable transmission rates. Expressions for these rates take into account the "cross-channels" established by the ISI/ICI and are based on lower bounds on mutual information that assume independent and identically distributed input data symbols. In agreement with recent statistical analyses of experimental shallow-water data, the channel is modeled as a multivariate Rician fading process with a slowly time-varying mean and with potentially correlated scatterers, which is more general than the common wide-sense stationary uncorrelated scattering model. Numerical assessments on real UA channels with spread factors around $10^{-1}$ show that reliable OFDM transmissions at 2 to $4 \mathrm{bits} / \mathrm{sec} / \mathrm{Hz}$ are achievable provided an average signal-to-noise ratio of 15 to $20 \mathrm{~dB}$.
\end{abstract}

\section{Introduction}

The various collections of underwater acoustic (UA) channels surveyed worldwide highlight the diversity of UA propagation environments and confirm in many cases the bad reputation of these channels as communication media [1-4]. UA communication systems are usually prone to time and frequency dispersion due to multipath propagation and Doppler effects, and the absorption of acoustic waves at high frequencies strongly limits their bandwidth. Finding systems that are robust to the environment, while maintaining acceptable data rates, remains the major difficulty faced by UA communication system designers. For a given propagation channel, this interplay between robustness and data rate results in practice in choosing different modulation schemes according to the importance we give to one or the other characteristic, the optimal trade-off being unknown in most cases. Historically, single-carrier modulations with receivers relying on channel equalizers in the time domain have been widely studied and used in practice for highspeed underwater communications [5]. Recently, multicarrier (MC) systems such as OFDM (orthogonal frequency division multiplexing) [6-9] have generated much interest due to the simplicity of receivers and the flexibility they offer.

For time-invariant channels, modulation-basis functions of common OFDM signals (e.g., cyclic prefix-based OFDM) can be seen as "eigen" functions of the channel operator and ensure the absence of interference at reception. This way of "diagonalizing" the channel allows the use of simple algorithms for recovering the information from the received 
signal. For double-dispersive channels, and particularly in the UA context, perfect channel diagonalization can rarely be achieved as the environment is generally random so that the channel eigenstructure differs from one channel realization to another. The channel diagonalization can then only be performed in some approximate sense [10-12] and interference due to time-frequency dispersion becomes inevitable. However, even if UA-OFDM systems can hardly avoid interference, compared to single-carrier approaches, multiplexing the information to be transmitted on a timefrequency grid offers the opportunity to optimize more degrees of freedom. OFDM system design is classically approached from the viewpoint of intersymbol/intercarrier interference (ISI/ICI) through maximization of the signalto-interference ratio (SIR) [10,11,13-15]. For a given set of channels, finding the MC signaling scheme that maximizes the average SIR is a way of designing robust systems that do not require complex equalization algorithms at reception. While the SIR may be a good figure of merit to assess the robustness of communications in doubly dispersive environments, it does not reveal the effect of the chosen signaling scheme on the information rate. The robustness improvement is generally paid back by the loss of spectral efficiency induced by the use of time and/or frequency guard intervals required to limit the interference. For instance, typical UA-OFDM systems use a guard interval between symbols that is lower-bounded by the maximum delay spread of the channel, which often lasts several tens of milliseconds. Compared to the active symbol duration, this interval is usually not negligible and can significantly reduce the transmission efficiency. Therefore, it remains unclear whether or not OFDM systems should tolerate slightly increased interference but operate at higher data rate.

Motivated by recent results in information theory and UA channel modeling, an information-theoretic analysis of the trade-off between maximization of SIR and minimization of guard intervals is proposed in this paper. More precisely, our main goal is to obtain a better understanding of the interplay between interference and the achievable transmission rate of UA-OFDM systems. We pay special attention to UA channels, where time and frequency dispersions are high enough so that ISI/ICI cannot be neglected in the information-theoretic treatment. Our analysis addresses two questions. First, what are the achievable rates of UA-OFDM in highly dispersive channels? And as a corollary, what are the consequences of OFDM design choices on these rates?

The target of our analysis is the investigation of UAOFDM information rate. To this end, we believe that the following aspects need to be accounted for:

(A1) the UA channel is selective both in time and frequency;

(A2) the UA channel cannot systematically be modeled as a wide-sense stationary uncorrelated scattering (WSSUS) process;

(A3) no perfect channel state information (CSI) is available at the transmitter nor the receiver;

(A4) interference is not negligible a priori.
These aspects are important as they may have a strong impact on the achievable rates of UA-OFDM systems. (A1) is particularly true in shallow-water environments where the spread factor (product between the delay and Doppler spread) is usually around $\left[10^{-3}, 10^{-1}\right]$ and can even exceed 1 in some cases [1]. The WSSUS assumption discussed in (A2) implies that the channel correlation function is timeinvariant and that the scatterers with different path delays are uncorrelated so that the second-order statistics of the channel are reduced from four to two dimensions [16]. While this assumption may be valid for data transmission at low bandwidth with static communication endpoints, it is not the case with moving platforms and/or when the path-loss associated with each channel path cannot be assumed to be constant over the transmission bandwidth $[2,17]$. (A3) corresponds to what is commonly referred to as the noncoherent setting where neither the transmitter nor the receiver knows the current realization of the channel perfectly [18-20]. This assumption has to be contrasted with the coherent setting where a genie provides the receiver with perfect CSI. (We warn the reader that the word coherent is here used in an information-theoretic context and its definition slightly differs from the one used in a demodulation context.) For most channels, the coherent model is not realistic since receivers are not genie-aided, and the effort to acquire the CSI usually induces some rate loss (pilots insertion, channel estimation errors, etc.). In addition, assuming perfect CSI at the transmitter is also optimistic for most practical cases since the low propagation speed of acoustic waves imposes strong constraints on the nature of the CSI provided by a feedback link. Since we focus on highly dispersive channels, we consider in (A4) that interference must be explicitly accounted for in the information theoretic-treatment.

To the best of our knowledge, the information rate of OFDM systems under such general assumptions has not yet been derived. However, recent works presented in [17] and more particularly in [21] give some useful ingredients to derive this rate. In [17], the authors derive bounds on the achievable rate of UA-OFDM systems and consider the aspects (A1) and (A3), and (A2) in part. Although correlated scattering is taken into account in their channel model, they assume wide-sense stationarity. As for interference, it is neglected in their analysis. In [21], Durisi et al. explicitly account for interference terms but present their results for WSSUS Rayleigh fading channels, which is not appropriate for the majority of UA channels $[2,22]$.

The main contributions of this paper are the following:

(i) based on the UA channel characterization presented in [2], we present an exact analysis of ISI/ICI of UA-OFDM systems transmitting in non-WSSUS channels. The channel is modeled as a multivariate Rician fading process with a slowly time-varying mean and with potentially correlated scatterers;

(ii) the information rate of UA-OFDM systems is analyzed under the general scenario described by the aspects (A1)-(A4); 
(iii) in order to extract guidelines useful for UA-OFDM system design, theoretical results are then numerically assessed on rectangular pulse shaping OFDM transmitting over experimental UA channels surveyed at sea.

This paper is organized as follows. Section 2 is devoted to the presentation of the system model and the main assumptions. Signal-to-interference ratio and achievable rates of OFDM systems transmitting over UA channels are derived in Section 3. In Section 4, we discuss the impact of channel and OFDM parameters on the information rate through various numerical experiments. Finally, conclusions are given in Section 5.

\section{System Model}

2.1. Notation. Throughout this paper, lowercase boldface letters denote vectors, for example, $\mathbf{x}$, and uppercase boldface letters denote matrices, for example, A. The superscripts ${ }^{T}$ and ${ }^{\dagger}$ denote transposition and Hermitian transposition, respectively. The Hadamard (elementwise) products of two matrices $\mathbf{A}$ and $\mathbf{B}$ is written as $\mathbf{A} \odot \mathbf{B}$. The entries of a matrix $\mathbf{A}$ are denoted by $[\mathbf{A}]_{k, n}$, where the indices $k$ and $n$ start at $0 . \mathbf{B}^{\left(k, k^{\prime}\right)}$ designates a submatrix of a block matrix $\mathbf{B}$. The Kronecker symbol is denoted by $\delta(k)$. We let $\operatorname{diag}(\mathbf{x})$ designate a diagonal square matrix whose main diagonal contains the elements of the vector $\mathbf{x}$. The inner product between two signals $y(t)$ and $z(t)$ is denoted by $\langle y, z\rangle=$ $\int_{-\infty}^{+\infty} y(t) z^{*}(t) d t$. $\mathcal{C} \mathcal{N}(\mathbf{m}, \mathbf{R})$ designates the distribution of a jointly proper Gaussian random vector [23] with mean $\mathbf{m}$ and covariance matrix R. Finally, $\mathbb{E}\{\cdot\}$ denotes expectation.

2.2. Channel Model. We consider a doubly selective baseband equivalent underwater acoustic channel, modeled as a random linear time-varying system 내 that maps input signals $x(t)$ into output signals $y(t)$ according to the $\mathrm{I} / \mathrm{O}$ relationship:

$$
y(t)=(\mathbb{H} x)(t)+w(t)=\int_{\tau} h_{\mathbb{U}}(\tau, t) x(t-\tau) d \tau+w(t),
$$

where $h_{\mathbb{q}}(\tau, t)$ is the channel impulse response, and $w(t)$ denotes the ambient noise.

According to recent results on the statistical characterization of UA channels [2], the impulse response is modeled as a trend stationary random process so that, for all $t, t_{1}$ and $t_{2} \in \mathbb{R}$

$$
h_{\mathbb{\sharp}}(\tau, t)=\tilde{h}_{\mathbb{U}}(\tau, t)+\bar{h}_{\mathbb{\sharp}}(\tau, t),
$$

with

$$
\begin{aligned}
& \mathbb{E}\left\{h_{\mathbb{\square}}(\tau, t)\right\}=\bar{h}_{\mathbb{\square}}(\tau, t), \\
& \mathbb{E}\left\{\left(h_{\mathbb{\sharp}}\left(\tau, t_{1}\right)-\mathbb{E}\left\{h_{\mathbb{\sharp}}\left(\tau, t_{1}\right)\right\}\right)\left(h_{\mathbb{\sharp}}\left(\tau, t_{2}\right)-\mathbb{E}\left\{h_{\mathbb{\sharp}}\left(\tau, t_{2}\right)\right\}\right)^{*}\right\} \\
& =\mathbb{E}\left\{\tilde{h}_{\llbracket \llbracket}\left(\tau, t_{1}\right) \tilde{h}_{\square-}^{*}\left(\tau, t_{2}\right)\right\} \\
& =\mathbb{E}\left\{\tilde{h}_{\mathbb{}}(\tau, t) \tilde{h}_{\mathbb{U}}^{*}\left(\tau, t+t_{2}-t_{1}\right)\right\}
\end{aligned}
$$

$\bar{h}_{\mathbb{H}}(\tau, t)$ is called the trend and is a slowly time-varying deterministic component. $\tilde{h}_{\mathbb{\sharp}}(\tau, t)$ is a zero-mean wide-sense stationary random process assumed to be Gaussian. This model describes the UA channel as a multivariate Rician fading process with a slowly time-varying mean. $\bar{h}_{\mathbb{\sharp}}(\tau, t)$ can be interpreted as the contribution of (pseudo) deterministic physical phenomena to channel fluctuations (wave undulation, range/depth dependence, bathymetry changes, etc.), and $\tilde{h}_{\llbracket \square}(\tau, t)$ represents the channel fluctuations attributable to scatterers that result in fast fading. Note that since no particular assumption is made about the correlation of scatterers, the model is very general and includes the WSSUS model as a subset. (Under the WSS assumption, the channel impulse response would satisfy (4) as well as $\bar{h}_{\mathbb{U}}\left(\tau, t_{1}\right)=\bar{h}_{\llbracket}\left(\tau, t_{2}\right), \forall t_{1}, t_{2} \in \mathbb{R}$. Under the assumption that $\mathbb{E}\left\{\tilde{h}_{\mathbb{⿴}}\left(\tau_{1}, t_{1}\right) \tilde{h}_{\square \mathbb{}}^{*}\left(\tau_{2}, t_{2}\right)\right\}=0$ for $\tau_{1} \neq \tau_{2}$, the channel would be said to exhibit delay uncorrelated scattering (US).) Without loss of generality, the channel is assumed to be normalized so that

$$
\lim _{T \rightarrow \infty} \frac{1}{T} \int_{-T / 2}^{T / 2} \int_{\tau} \mathbb{E}\left\{\left|h_{\mathbb{U}}(\tau, t)\right|^{2}\right\} d \tau d t=1 .
$$

We define the channel Rice factor as the power ratio between the deterministic trend and the random component, that is,

$$
\kappa=\lim _{T \rightarrow \infty} \frac{1}{T} \frac{\int_{-T / 2}^{T / 2} \int_{\tau}\left|\bar{h}_{\mathbb{\boxplus}}(\tau, t)\right|^{2} d \tau d t}{\int_{\tau} \mathbb{E}\left\{\left|\tilde{h}_{\mathbb{\square}}(\tau, t)\right|^{2}\right\} d \tau} .
$$

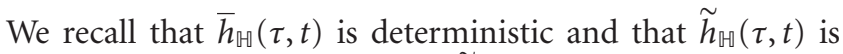
wide-sense stationary so that $\mathbb{E}\left\{\left|\tilde{h}_{\mathbb{}}(\tau, t)\right|^{2}\right\}$ does not depend on $t$.

The ambient noise $w(t)$ is assumed to be Gaussian and to result from the mixture of four sources [24]: turbulence, shipping, waves, and thermal noise with nonflat power spectral densities (PSDs). We, therefore, modeled $w(t)$ as a nonwhite zero-mean wide-sense stationary Gaussian random process with correlation function:

$$
R_{w}\left(t_{2}-t_{1}\right) \triangleq \mathbb{E}\left\{w\left(t_{1}\right) w\left(t_{2}\right)^{*}\right\}
$$

and PSD

$$
W(f) \triangleq \int_{\tau} R_{w}(\tau) e^{-j 2 \pi \tau f} d \tau
$$

Simple approximated models for $R_{w}(\tau)$ and $W(f)$ are given in Appendix A. 
In addition to the channel impulse response, another channel function that will be important for our treatment is the delay-Doppler spreading function:

$$
\begin{aligned}
& G_{\mathbb{\sharp}}(\tau, v)=\int_{-\infty}^{\infty} h_{\mathbb{\sharp}}(\tau, t) e^{-2 j \pi v t} d t \\
& =\underbrace{\int_{-\infty}^{\infty} \tilde{h}_{\mathbb{\sharp}}(\tau, t) e^{-2 j \pi v t} d t}_{\triangleq \widetilde{G}_{\mathbb{H}}(\tau, v)} \\
& +\underbrace{\int_{-\infty}^{\infty} \bar{h}_{\mathbb{H}}(\tau, t) e^{-2 j \pi v t} d t}_{\triangleq \bar{G}_{\mathbb{H}}(\tau, \nu)} .
\end{aligned}
$$

The channel I/O relation (1) can now be written as

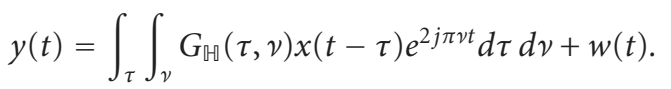

The spreading function is assumed to be compactly supported on a rectangle and satisfies

$$
\begin{gathered}
G_{\mathbb{\square}}(\tau, \nu)=0, \\
\text { for }(\tau, \nu) \notin\left[0, \tau_{\max }\right] \times\left[-\frac{\nu_{\max }}{2} ; \frac{\nu_{\max }}{2}\right],
\end{gathered}
$$

where $\tau_{\max }$ and $\nu_{\max }$ denote the maximum time delay spread and the maximum Doppler spread, respectively. This assumption leads to the following definition of the channel spread factor:

$$
\Delta_{\mathbb{q}} \triangleq \tau_{\max } \times \nu_{\max }
$$

Note that this assumption eases the analysis proposed in this paper but is only an approximation of real channels behavior. In practice, to set values to $\tau_{\max }$ and $\nu_{\max }$, it is often required to resort to more empirical definitions (e.g., threshold-based definitions, as used in Section 4.2). Various definitions of delay and Doppler spreads for real channels are discussed in [1, Section 4.5].

2.3. OFDM Signal. OFDM signaling schemes can be described by two Weyl-Heisenberg (WH) sets $[10,11,13]$ : the one used at transmission, expressed as

$$
(g, T, F) \triangleq\left\{g_{k, n}(t)=g(t-k T) e^{j 2 \pi n F t},\|g\|^{2}=1\right\}_{k, n \in \mathbb{Z}}
$$

and the one used at reception, defined as

$$
(\gamma, T, F) \triangleq\left\{\gamma_{k, n}(t)=\gamma(t-k T) e^{j 2 \pi n F t},\|\gamma\|^{2}=1\right\}_{k, n \in \mathbb{Z}},
$$

where $T, F>0$ are the time and frequency shifts of the prototype function $g(t)$ and $\gamma(t)$. The signaling scheme is here assumed to be (bi)orthogonal, so that

$$
\left\langle g_{k, n}, \gamma_{k^{\prime}, n^{\prime}}\right\rangle=\delta\left(k-k^{\prime}\right) \delta\left(n-n^{\prime}\right) \text {. }
$$

To ease the readability of the results presented in the sequel, we shall restrict our analysis to orthogonal receive pulses (i.e., $\left.\left\langle\gamma_{k, n}, \gamma_{k^{\prime}, n^{\prime}}\right\rangle=\delta\left(k-k^{\prime}\right) \delta\left(n-n^{\prime}\right)\right)$. (Note that nonorthogonal receive pulses introduce noise correlation and noiseenhancement that can be harmful for advanced equalization techniques. As an example, cyclic prefix based OFDM receiver are orthogonal whereas zero-padding receivers (with $T F>1$ ) are not.)

The transmitted signal is

$$
x(t)=\sum_{k=0}^{K-1} \sum_{n=0}^{N-1} x_{k, n} g_{k, n}(t),
$$

where $N$ is the number of subcarriers, and $K T$ is the approximate duration of the transmitted signal. $x_{k, n}$ denotes the data symbols. Since little is known about the exact structure of optimal signaling under the general constraints listed in the introduction, we restrict our analysis to zero-mean, independent and identically distributed (i.i.d.) symbols. We assume that the average power of the input signals is limited so that

$$
\frac{1}{K T} \sum_{k=0}^{K-1} \sum_{n=0}^{N-1} \mathbb{E}\left\{\left|x_{k, n}\right|^{2}\right\}=P,
$$

where $P<+\infty$ is the maximum average power available. The signal-to-noise ratio (SNR) is then defined as

$$
\rho \triangleq \frac{P}{\int_{0}^{B} W(f)},
$$

where $B=N F$ denotes the system bandwidth.

At reception, the output signal $y(t)$ is projected onto the set $\left\{\gamma_{k, n}(t)\right\}$ to obtain

$$
y_{k, n} \triangleq\left\langle y, \gamma_{k, n}\right\rangle=\left\langle\uplus x, \gamma_{k, n}\right\rangle+\underbrace{\left\langle w, \gamma_{k, n}\right\rangle}_{\triangleq_{w_{k, n}}} .
$$

$y_{k, n}$ can be developed as

$$
\begin{aligned}
y_{k, n}= & \left\langle\mathbb{H} g_{k, n}, \gamma_{k, n}\right\rangle x_{k, n} \\
& +\sum_{\substack{k^{\prime}=0 n^{\prime}=0 \\
\left(k^{\prime}, n^{\prime}\right) \neq(k, n)}}^{K-1}\left\langle\mathbb{H} g_{k^{\prime}, n^{\prime}}, \gamma_{k, n}\right\rangle x_{k^{\prime}, n^{\prime}}+w_{k, n},
\end{aligned}
$$

where the second term on the right-hand side (RHS) of (20) represents the intersymbol and intercarrier interference.

The relation (20) can be compactly expressed as

$$
\mathbf{y}=\mathbf{H x}+\mathbf{w}
$$

where the channel input and output vectors of size $N K \times 1$ are respectively defined by

$$
\begin{aligned}
& \mathbf{x} \triangleq\left[\begin{array}{llll}
\mathbf{x}_{0}^{T} & \mathbf{x}_{1}^{T} \cdots \mathbf{x}_{K-1}^{T}
\end{array}\right]^{T}, \\
& \text { with } \mathbf{x}_{k} \triangleq\left[x_{k, 0} x_{k, 1} \cdots x_{k, N-1}\right]^{T} \text {, } \\
& \mathbf{y} \triangleq\left[\begin{array}{llll}
\mathbf{y}_{0}^{T} & \mathbf{y}_{1}^{T} & \cdots & \mathbf{y}_{K-1}^{T}
\end{array}\right]^{T}, \\
& \text { with } \mathbf{y}_{k} \triangleq\left[\begin{array}{llll}
y_{k, 0} & y_{k, 1} & \cdots & y_{k, N-1}
\end{array}\right]^{T} \text {, }
\end{aligned}
$$


and where $\mathbf{w}$ is defined analogously. The $N K \times N K$ channel matrix $\mathbf{H}$ is given by

$$
\mathbf{H} \triangleq\left(\begin{array}{ccc}
\mathbf{H}^{(0,0)} & \cdots & \mathbf{H}^{(0, K)} \\
\vdots & \ddots & \vdots \\
\mathbf{H}^{(K, 0)} & \cdots & \mathbf{H}^{(K, K)}
\end{array}\right),
$$

where the matrix block $\mathbf{H}^{\left(k, k^{\prime}\right)}$ of size $N \times N$ satisfies

$$
\left[\mathbf{H}^{\left(k, k^{\prime}\right)}\right]_{n, n^{\prime}} \triangleq\left\langle\mathbb{M} g_{k^{\prime}, n^{\prime}}, \gamma_{k, n}\right\rangle .
$$

Since we do not neglect interference, the matrix $\mathbf{H}$ is not diagonal and can be decomposed as follows:

$$
\mathbf{H}=\operatorname{diag}(\mathbf{h})+\mathbf{Z},
$$

where $\mathbf{h}$ is the direct channel vector corresponding to the main diagonal of $\mathbf{H}$ and $\mathbf{Z}$ is the ISI/ICI cross-channel matrix containing the offdiagonal terms of $\mathbf{H}$.

\section{Information Theoretic Analysis of UA-OFDM Systems}

Three fundamental characteristics of the sets $(g, T, F)$ and $(\gamma, T, F)$ are generally involved in the optimization/ performance of MC systems:

(i) (bi)orthogonality: for an ideal channel where $y(t)=$ $x(t)$, perfect demodulation is obtained iff $g(t)$, and $\gamma(t)$ satisfies the condition (15);

(ii) localization: localization of a prototype function involves the Heisenberg uncertainty principle and characterizes its time-frequency concentration so that it directly affects the power of interference observed at reception;

(iii) density: spectral efficiency of MC systems is directly proportional to the density $1 / T F$ of the timefrequency grid that supports the transmission scheme. For instance, adding guard intervals between OFDM symbols reduces the density due to the fact that $T F>1$ in this case, but as the product TF gets larger, the power of ISI/ICI diminishes.

Ideally, we would like to construct a MC system that is (bi)orthogonal, with well-localized prototype functions (to limit the interference) and with a dense time-frequency grid (to maximize the spectral efficiency). However, these three conditions cannot be satisfied simultaneously due to the Balian-Low theorem [25, Th. 4.1.1.] More precisely, welllocalized (bi)orthogonal pulses can only be found for $T F>$ 1 (see [26, Ch. 2] for more details). This loss in spectral efficiency is usually the price to pay to mitigate ISI/ICI over double-dispersive channels. (Note that other approaches, e.g. [13] or [27], privilege localization and spectral efficiency over (bi)orthogonality.)

These elements highlight the difficulty of finding a compromise between a low interference at reception and a maximal use of the degrees of freedom offered by the channel. The optimal trade-off between low interference and high spectral efficiency is a key ingredient in OFDM system design that has yet to be found. To provide some guidelines that will help us to progress toward the optimal solution, we suggest to study the signal-to-interference ratio as well as the information rate of UA-OFDM systems. The information rate, defined as the amount of information that can be transmitted with arbitrarily small error probability, appears as a good figure of merit for system design as it jointly considers interference and spectral efficiency.

3.1. Signal-to-Interference Ratio. The signal-to-interference ratio at the symbol $k$ and the subcarrier $n$ is defined as

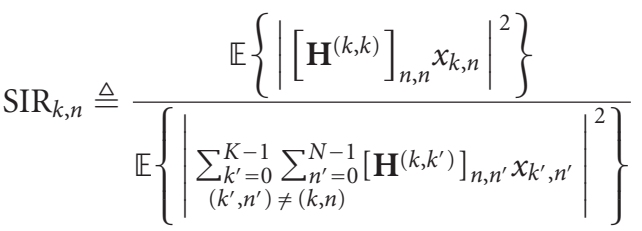

$$
\begin{aligned}
& \stackrel{\text { (a) }}{=} \frac{\mathbb{E}\left\{\left|\left[\mathbf{H}^{(k, k)}\right]_{n, n}\right|^{2}\right\}}{\sum_{\substack{k^{\prime}=0 \\
\left(k^{\prime}, n^{\prime}\right) \neq(k, n)}}^{K-1} \sum_{n^{\prime}=0}^{N-1} \mathbb{E}\left\{\left|\left[\mathbf{H}^{\left(k, k^{\prime}\right)}\right]_{n, n^{\prime}}\right|^{2}\right\}},
\end{aligned}
$$

where (a) follows from the assumption that the $x_{k, n}$ are i.i.d. with zero mean. The numerator represents the average power of the diagonal entries of $\mathbf{H}$, and the denominator the power of its offdiagonal entries. Let $A_{g, \gamma}(\tau, \nu)$ be the crossambiguity function of $g(t)$ and $\gamma(t)$ defined as follows:

$$
A_{g, \gamma}(\tau, v) \triangleq \int_{t} g(t) \gamma^{*}(t-\tau) e^{-j 2 \pi v t} d t .
$$

The signal as well as the interference power can be expressed as a function of $A_{g, \gamma}(\tau, \nu)$. More precisely, as shown in Appendix B:

$$
\begin{aligned}
& \mathbb{E}\left\{\left|\left[\mathbf{H}^{(k, k)}\right]_{n, n}\right|^{2}\right\} \\
& \approx \int_{\tau} \int_{\tau^{\prime}} \int_{\nu} \widetilde{S}_{\mathbb{H}}\left(\tau, \tau^{\prime}, \nu\right) A_{\gamma, g}^{*}(\tau, \nu) \\
& \quad \times A_{\gamma, g}\left(\tau^{\prime}, \nu\right) e^{j 2 \pi n F\left(\tau^{\prime}-\tau\right)} d \nu d \tau^{\prime} d \tau \\
& \quad+\left|\int_{\tau} \bar{h}_{\mathbb{H}}(\tau, k T) A_{\gamma, g}^{*}(\tau, 0) e^{-j 2 \pi n F \tau} d \tau\right|^{2},
\end{aligned}
$$

where $\widetilde{S}_{\mathbb{H}}\left(\tau, \tau^{\prime}, v\right)$ is the channel scattering function defined as

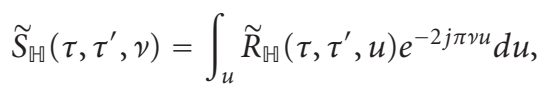

with $\widetilde{R}_{\mathbb{\sharp}}\left(\tau, \tau^{\prime}, u\right) \triangleq \mathbb{E}\left\{\tilde{h}_{\mathbb{}}(\tau, t) \tilde{h}_{\mathbb{\sharp}}^{*}\left(\tau^{\prime}, t+u\right)\right\}$. Note that in the case where the scatterers are assumed to be uncorrelated, the scattering function is simplified to $\widetilde{S}_{\mathbb{\sharp}}\left(\tau, \tau^{\prime}, \nu\right) \delta\left(\tau^{\prime}-\tau\right)$.

The first term on the RHS of (28) represents the power carried by the fast fading random part of the channel, 
and the second term corresponds to the deterministic part of the channel. Similarly, the interference power satisfies

$$
\sum_{\substack{k^{\prime}=0 \\\left(k^{\prime}, n^{\prime}\right) \neq(k, n)}}^{K-1} \sum_{n^{\prime}=0}^{N-1} \mathbb{E}\left\{\left|\left[\mathbf{H}^{\left(k, k^{\prime}\right)}\right]_{n, n^{\prime}}\right|^{2}\right\} \triangleq \tilde{\sigma}_{I_{n}}^{2}+\bar{\sigma}_{I_{k, n}}^{2},
$$

where $\tilde{\sigma}_{I_{n}}^{2}$ is the interference power corresponding to the random part of the channel and is expressed as

$$
\begin{aligned}
\tilde{\sigma}_{I_{n}}^{2}=\sum_{\substack{k^{\prime}=1-K \\
\left(k^{\prime}, n^{\prime}\right) \neq(0,0)}}^{K-1} \sum_{n^{\prime}=1-N}^{N-1} \int_{\tau} \int_{\tau^{\prime}} \int_{\nu} & \widetilde{S}_{\mathfrak{⿴}}\left(\tau, \tau^{\prime}, \nu\right) \\
& \times A_{\gamma, g}^{*}\left(\tau+k^{\prime} T, \nu+n^{\prime} F\right) \\
& \times A_{\gamma, g}\left(\tau^{\prime}+k^{\prime} T, \nu+n^{\prime} F\right) \\
& \times e^{j 2 \pi F\left(n^{\prime}+n\right)\left(\tau^{\prime}-\tau\right)} d \nu d \tau^{\prime} d \tau .
\end{aligned}
$$

Note that $\tilde{\sigma}_{I_{n}}^{2}$ does not depend on $k$ since, according to (4), $\tilde{h}_{\mathbb{U}}(\tau, t)$ is wide-sense stationary.

$\bar{\sigma}_{I_{k, n}}^{2}$ is the interference power due to the deterministic part of the channel and is given by

$$
\begin{array}{r}
\bar{\sigma}_{I_{k, n}}^{2}=\sum_{\substack{k^{\prime}=1-K n^{\prime}=1-N \\
\left(k^{\prime}, n^{\prime}\right) \neq(0,0)}}^{K-1} \mid \int_{\tau} \bar{h}_{\mathbb{\tau}}\left(\tau,\left(k^{\prime}+k\right) T\right) \\
\times A_{\gamma, g}^{*}\left(\tau+k^{\prime} T, n^{\prime} F\right) \\
\\
\left.\quad e^{-j 2 \pi F\left(n^{\prime}+n\right) \tau} d \tau\right|^{2} .
\end{array}
$$

Using the above quantities, we now define the average signal-to-interference ratio as

$$
\overline{\mathrm{SIR}} \triangleq \lim _{K \rightarrow \infty} \frac{1}{K N} \sum_{k=0}^{K-1} \sum_{n=0}^{N-1} \operatorname{SIR}_{k, n} .
$$

3.2. Achievable Rate. Let $\mathcal{P}_{\mathbf{x}}$ be the set of probability distributions on $\mathbf{x}$ that satisfy the constraints given in (17). The maximum achievable rate for an OFDM system is then given by [28]

$$
C=\lim _{K \rightarrow \infty} \frac{1}{K T} \sup _{P_{\mathbf{x}}} I(\mathbf{y} ; \mathbf{x}),
$$

where $I(\mathbf{y} ; \mathbf{x})=h_{E}(\mathbf{y})-h_{E}(\mathbf{y} \mid \mathbf{x})$ is the mutual information between $\mathbf{y}$ and $\mathbf{x}$ with $h_{E}(\mathbf{y})$, the differential entropy of $\mathbf{y}$. In the noncoherent setting, the maximum achievable rate is notoriously hard to characterize analytically. However, by evaluating the mutual information $I(\mathbf{y} ; \mathbf{x})$ for a specific input distribution, and by relying on the following inequality on mutual information [29]:

$$
I(\mathbf{y} ; \mathbf{x}) \geq I(\mathbf{y} ; \mathbf{x} \mid \mathbf{H})-I(\mathbf{y} ; \mathbf{H} \mid \mathbf{x})
$$

we can get a lower bound on $C$ that yields an informationtheoretic criterion useful for the analysis of UA-OFDM systems. Note that the first term on the right-hand side of (35) corresponds to the coherent information rate under perfect channel knowledge at reception, and the second term can be interpreted as a penalty term that quantifies the rate loss due to the lack of channel knowledge.

Theorem 1. The maximum achievable rate of an OFDM system with i.i.d. input symbols satisfying the average-power constraint (17) and transmitting over the channel modeled by (2) is lower-bounded as $C^{L 1} \leq C$, where

$$
\begin{aligned}
C^{L 1}=\lim _{K \rightarrow \infty} \frac{1}{K T} \mathbb{E}_{\mathbf{H}}\left\{\log \operatorname{det}\left(\mathbf{I}+\frac{P T}{N} \mathbf{H H}^{\dagger} \operatorname{diag}\left(\mathbf{r}_{w}\right)^{-1}\right)\right\} \\
-\inf _{0<\alpha<1} \frac{1}{K T}\left[\log \operatorname{det}\left(\mathbf{I}+\frac{P T}{N \alpha} \mathbf{R}_{\mathbf{h}} \operatorname{diag}\left(\mathbf{r}_{w}\right)^{-1}\right)\right. \\
\left.+K \sum_{n=0}^{N-1} \log \left(1+\frac{P T}{N(1-\alpha) r_{w}(n)} \tilde{\sigma}_{I_{n}}^{2}\right)\right] .
\end{aligned}
$$

Here, the entries of the $N K \times 1$ noise power vector $\mathbf{r}_{w}$ are defined as

$$
r_{w}(n+k K) \triangleq W(n F), \quad n \in[0, N-1], \quad k \in[0, K-1],
$$

and $\mathbf{R}_{\mathbf{h}}$ denotes the covariance matrix of the direct channel vector $\mathbf{h}$, whose entries are expressed as

$$
\begin{array}{r}
{\left[\mathbf{R}_{\mathbf{h}}^{\left(k, k^{\prime}\right)}\right]_{n, n^{\prime}}=\int_{\tau} \int_{\tau^{\prime}} \int_{\nu} \widetilde{S}_{\mathbb{\sharp}}\left(\tau, \tau^{\prime}, \nu\right) A_{\gamma, g}^{*}(\tau, \nu) A_{\gamma, g}\left(\tau^{\prime}, \nu\right)} \\
\quad \times e^{j 2 \pi \nu T\left(k-k^{\prime}\right)} e^{j 2 \pi F\left(n^{\prime} \tau^{\prime}-n \tau\right)} d \nu d \tau^{\prime} d \tau .
\end{array}
$$

Proof. See Appendix C.

Note that the penalty term in (36) only depends on the random component of the channel so that acquiring CSI at reception gets more costly as the channel gets more fluctuating (e.g., estimating $\mathbf{H}$ gets more difficult as the power of its off-diagonal entries increases).

To get a better insight into the achievable rate, the following corollary presents a simplified scenario of transmission that leads to a more tractable expression of the lower bound.

Corollary 2. In the case where the noise is assumed to be white and the scatterers uncorrelated, the maximum achievable rate is lower bounded as $C^{L 2} \leq C$, where

$$
\begin{array}{r}
C^{L 2}=\lim _{K \rightarrow \infty} \frac{1}{K T} \mathbb{E}_{\mathbf{H}}\left\{\log \operatorname{det}\left(\mathbf{I}+\rho T F \mathbf{H H}^{\dagger}\right)\right\} \\
-\inf _{0<\alpha<1} \frac{B}{T F}\left[\int_{-1 / 2}^{1 / 2} \log \left(1+\frac{\rho T F}{\alpha} \widetilde{s}(\theta)\right) d \theta\right. \\
\left.+\log \left(1+\frac{\rho T F}{1-\alpha} \tilde{\sigma}_{I}^{2}\right)\right] .
\end{array}
$$


Here, $\rho$ denotes the SNR defined in (18), $\widetilde{s}(\theta)$ is the PSD of the zero-mean stationary channel process $\left\{h_{k, n}-\mathbb{E}\left\{h_{k, n}\right\}\right\}_{k}$, and is expressed as

$$
\widetilde{\boldsymbol{s}}(\theta)=\sum_{k=-\infty}^{\infty} \int_{\tau} \int_{\nu} \tilde{S}_{\varpi \mathbb{}}(\tau, \nu)\left|A_{\gamma, g}(\tau, \nu)\right|^{2} e^{j 2 \pi v k T} d \nu d \tau e^{-j 2 \pi k \theta},
$$

and $\tilde{\sigma}_{I}^{2}$ satisfies

$$
\begin{aligned}
\tilde{\sigma}_{I}^{2}=\sum_{\substack{k^{\prime}=1-K n^{\prime}=1-N \\
\left(k^{\prime}, n^{\prime}\right) \neq(0,0)}}^{K-1} \int_{\tau} \int_{\nu} \tilde{S}_{\boxplus}(\tau, \nu) \\
\\
\times\left|A_{\gamma, g}^{*}\left(\tau+k^{\prime} T, \nu+n^{\prime} F\right)\right|^{2} d \nu d \tau .
\end{aligned}
$$

Proof. See Appendix D.

Note that the scenario depicted in Corollary 2 may be acceptable for systems transmitting in small bandwidth (on the order of $\mathrm{kHz}$ ), where the noise PSD can be assumed flat and where the propagation loss associated with each channel path is approximately constant over the transmit bandwidth, thus reducing the correlation between channel arrival paths.

\section{Numerical Analysis}

We next examine the signal-to-interference ratio and the bounds of the previous section in various scenarios. Using a synthetic channel model, impact of time-frequency dispersion on the information rate is first discussed in Section 4.1. Bounds on the information rate applied to experimental double-dispersive UA channels surveyed at sea are then analyzed in Section 4.2. Common OFDM systems with rectangular pulse shaping are used as a framework in our investigation. $g(t)$ and $\gamma(t)$ are thus defined as

$$
\begin{gathered}
g(t)= \begin{cases}\frac{1}{\sqrt{T}} & \text { if } 0<t \leq T, \\
0 & \text { otherwise, }\end{cases} \\
\gamma(t)= \begin{cases}\frac{1}{\sqrt{T-T_{g}}} & \text { if } T_{g}<t \leq T, \\
0 & \text { otherwise, }\end{cases}
\end{gathered}
$$

where $T_{g}=T-1 / F$ denotes the guard time between OFDM symbols.

4.1. Synthetic Channel Model. To illustrate the impact of channel dispersion on the performance of OFDM systems, we first consider a canonical channel model. It has no particular physical justification, but mimics a bad scenario from the viewpoint of a communication system [20] and will help us to provide general trends on OFDM system robustness against channel dispersion.

We assume the following environment:

(i) Rayleigh fading, that is, $\kappa=0$;

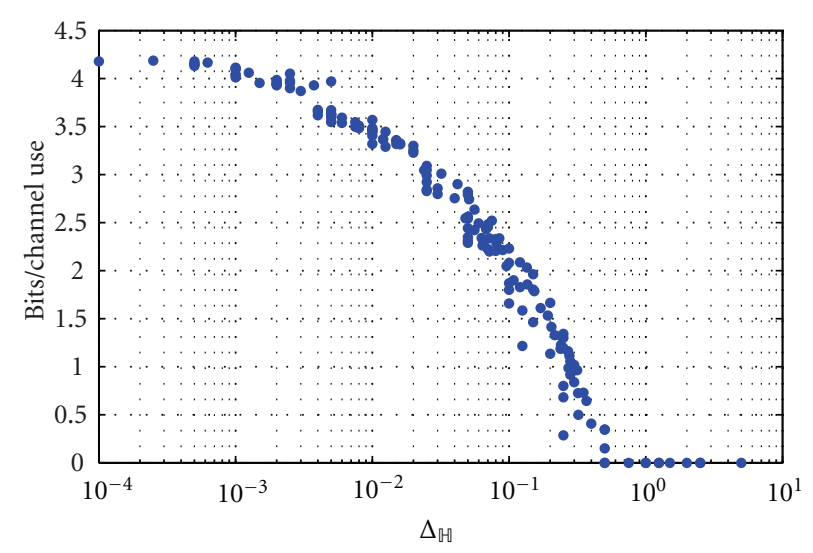

Figure 1: Lower bound (39) as a function of the channel spread factor for a Rayleigh fading channel with a brick-shaped scattering function and white Gaussian Noise. $\rho=15 \mathrm{~dB}$ and $B=5 \mathrm{kHz}$.

(ii) uncorrelated scatterers with a brick-shaped scattering function $\widetilde{S}_{\sharp}(\tau, \nu)=1 / \Delta_{\mathbb{H}}$;

(iii) white Gaussian noise.

Figure 1 shows the information rate as a function of the channel spread factor $\Delta_{\bullet}$ defined in (12). The grid parameters $T$ and $F$ are chosen according to the gridmatching rule [30]: $T / F=\tau_{\max } / \nu_{\max }$, and $T F$ is chosen so as to maximize (39). $\rho$ is set to $15 \mathrm{~dB}$ and $B=5 \mathrm{kHz}$. $\tau_{\max }$ is randomly chosen between 1 and $50 \mathrm{~ms}$, and $\nu_{\max }$ between 0.1 and $50 \mathrm{~Hz}$. As expected, the achievable rate of OFDM systems is strongly affected by both the delay and the Doppler spread and decreases as the channel gets more dispersive. Note that as the rectangular prototype function is not equally localized in time and frequency, there is not a single performance point for a given spread factor. For $\Delta_{\mathbb{H}} \leq 10^{-3}$ and $\rho=15 \mathrm{~dB}$, OFDM systems should be able to communicate at roughly $4 \mathrm{bits} / \mathrm{s} / \mathrm{Hz}$, which represents $80 \%$ of the achievable rate in an AWGN channel at the same SNR. Such systems can be relatively efficient as long as $\Delta_{\text {田 }}<10^{-1}$. However, for $\Delta_{\mathbb{H}}>1$, there is no guarantee that any data can be reliably transmitted.

4.2. Experimental UA Channels Recorded at Sea. Three different shallow water channels, recorded in the Atlantic ocean and the Mediterranean sea, are considered. Table 1 summarizes the main characteristics of these channels, and Figure 2 shows the evolution of their respective power delay profiles as a function of time. Channel (a) results from data collected by the DGA-TN (Direction Générale de l'Armement-Techniques Navales) in the Atlantic ocean off Brest (France) in October 2007, and channels (b) and (c) result from sea trials performed by Thales Underwater Systems in the Mediterranean sea off La Ciotat (France) in October 2004. From the raw data and for each channel, the trend $\bar{h}_{\mathbb{H}}(\tau, t)$ is separated from the random component $\tilde{h}_{\sharp}(\tau, t)$ using the empirical mode decomposition method [2]. The maximum time delay spread is estimated as the difference between the longest and the shortest delay, where 
TABLE 1: Summary of at sea experiments.

\begin{tabular}{|c|c|c|c|c|c|c|c|}
\hline Channel & $\begin{array}{c}\text { Center frequency } \\
(\mathrm{kHz})\end{array}$ & $\begin{array}{c}\text { Bandwidth } \\
(\mathrm{kHz})\end{array}$ & $\begin{array}{c}\text { Distance } \\
(\mathrm{m})\end{array}$ & $\begin{array}{l}\text { Water depth } \\
(\mathrm{m})\end{array}$ & $\begin{array}{c}\tau_{\max } \\
(\mathrm{ms})\end{array}$ & $\begin{array}{l}v_{\max } \\
(\mathrm{Hz})\end{array}$ & $\begin{array}{c}\kappa \\
(\mathrm{dB}) \\
\end{array}$ \\
\hline (a) & 17.5 & 2.9 & 1000 & $10-40$ & 2.1 & 1.5 & 15.5 \\
\hline (b) & 6 & 1 & 2500 & $60-120$ & 35 & 2.7 & 4.9 \\
\hline (c) & 6 & 1 & 5000 & $60-120$ & 47 & 3.2 & 1.6 \\
\hline
\end{tabular}

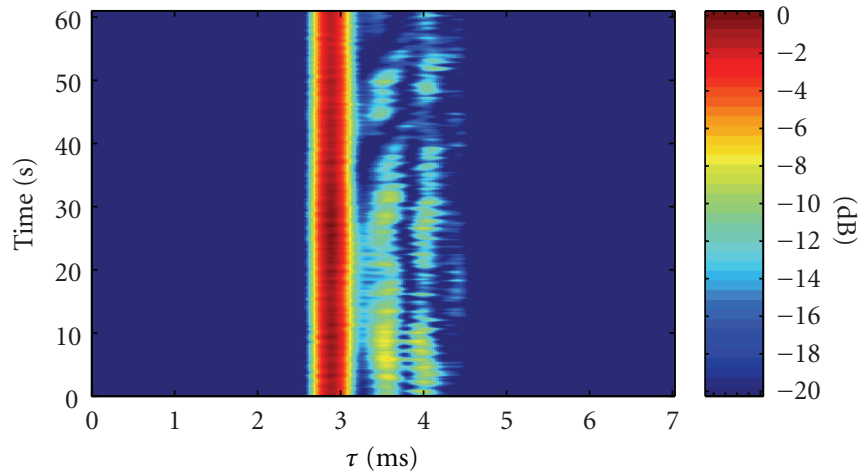

(a)

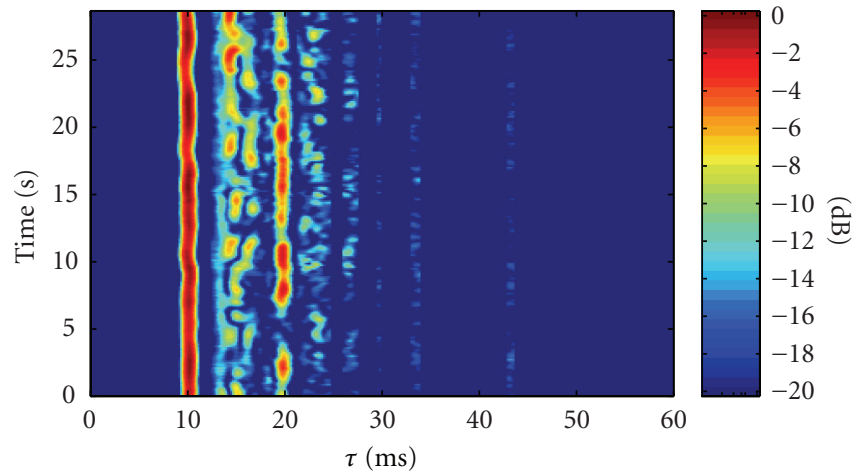

(b)

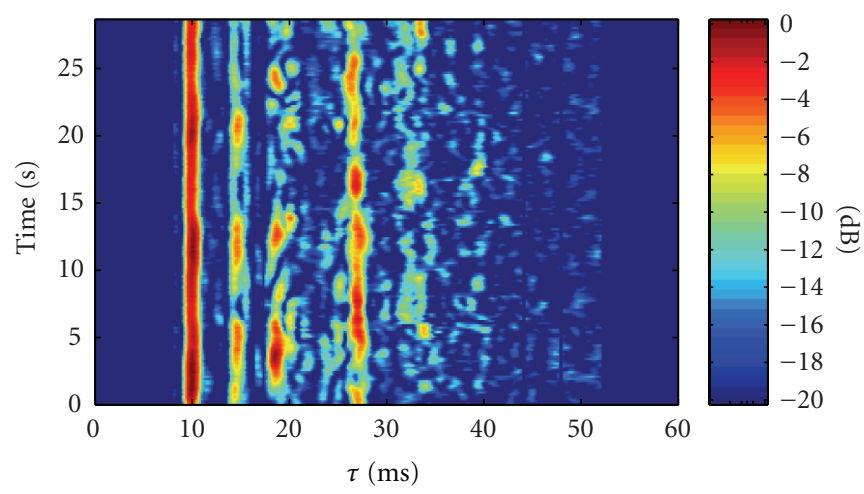

(c)

Figure 2: Time evolution of the power delay profiles of the channels depicted in Table 1.

the average power delay profile exceeds $1 \%$ of its maximum value (i.e., taps that are $20 \mathrm{~dB}$ below the strongest tap are assumed to result from noise and are artificially set to $0)$. The scattering function $\widetilde{S}_{\mathbb{U}}\left(\tau, \tau^{\prime}, \nu\right)$ is obtained from a correlogram estimate of PSD. The maximum Doppler spread is similarly defined from the Doppler power spectrum as the maximum delay spread from the average power delay profile. (The Doppler power spectrum is defined as $\int_{\tau} \int_{\tau^{\prime}} \widetilde{S}_{\mathfrak{t} \mathbb{}}\left(\tau, \tau^{\prime}, v\right) d \tau^{\prime} d \tau$.) To compute the various expectations required to evaluate the bound $C^{L 1}$, a large number of channel realizations are generated using the channel stochastic replay approach presented in [2]. Throughout this analysis, $T$ and $F$ satisfy the grid-matching rule mentioned previously (i.e., $T / F=\tau_{\max } / \nu_{\max }$ ).

In Figure 3, the average SIR is plotted as a function of $T F$ for the three channels. It can be noticed that as $T F$ increases, the duration of the guard interval increases as well, which results in a lower interference at reception. The SIR increases

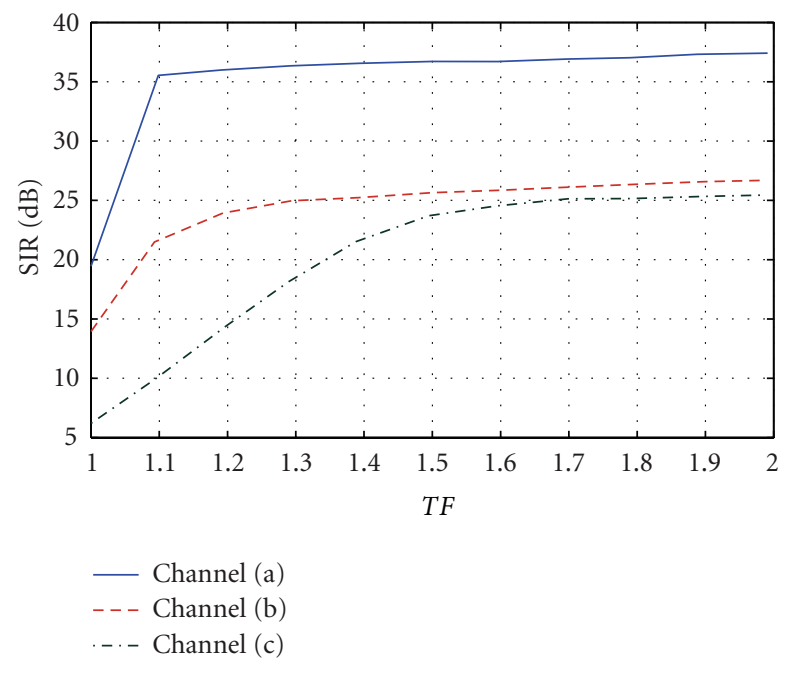

FIGURE 3: Average signal-to-interference ratio as a function of TF. 


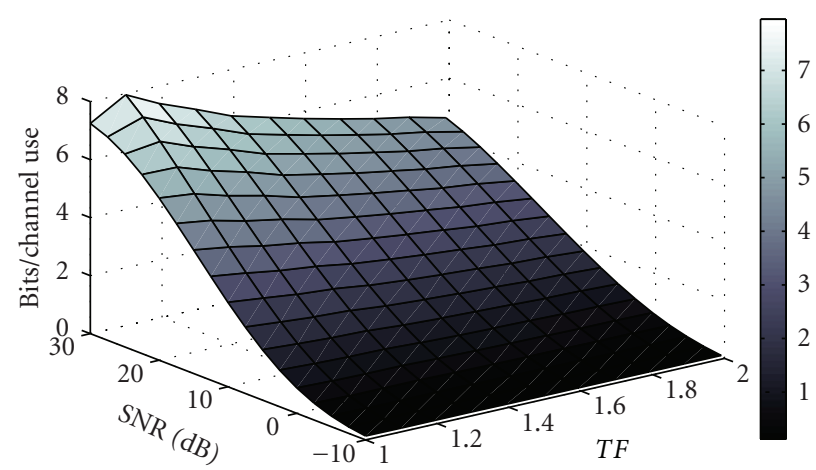

(a)

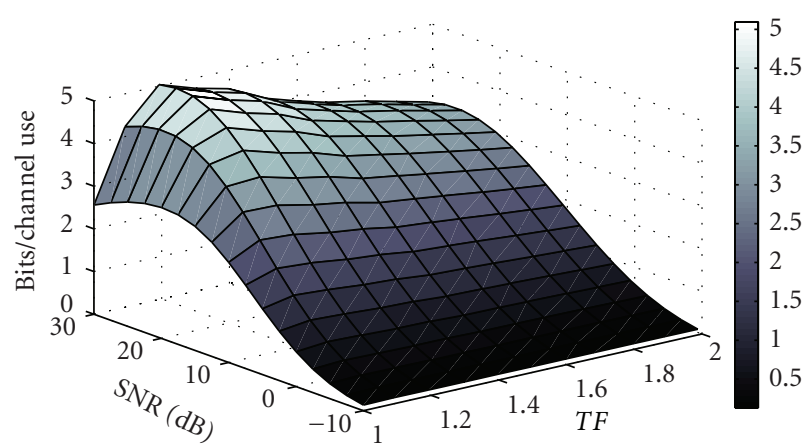

(b)

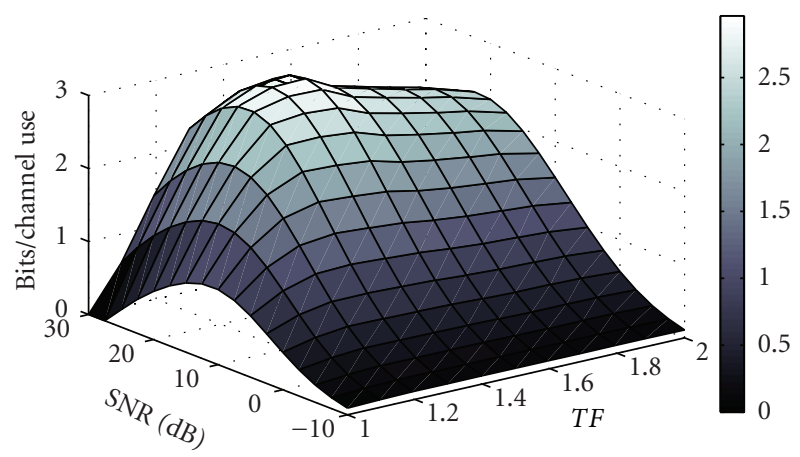

(c)

FIgURE 4: Lower-bound $C^{L 1}$ as a function of $T F$ and the SNR $\rho$ for the three channels depicted in Table 1.

significantly as long as $T F$ is such that the guard interval duration is lower than the maximum time delay spread. A further increase of TF produces a slighter increase of the SIR, which indicates that ISI is more detrimental than ICI. The average SIR also depends on the channel properties. As expected, for a given $T F$, the larger the spread factor, the smaller the SIR.

Through the evolution of the achievable rate (36) as a function of $T F$ and the SNR, Figure 4 shows possible tradeoffs between interference minimization and loss of signalspace dimensions. It provides a measure of reassurance that current practice in designing OFDM systems for underwater channels is reasonable. That is, oversizing guard intervals duration (i.e., choosing large $T F$ ) compared to the channel maximum delay spread is not much detrimental to the information rate, whereas a too small TF can significantly decreases this rate, especially in highly dispersive channels such as channel (c). The results of Figure 4 also suggest that significant rate improvements are possible compared to state-of-the-art UA-OFDM systems. For instance, in channels (b) and (c), reliable OFDM transmissions at 2 to $4 \mathrm{bits} / \mathrm{sec} / \mathrm{Hz}$ are achievable provided an average signalto-noise ratio of 15 to $20 \mathrm{~dB}$, whereas in the same SNR range, single-input single-output UA-OFDM systems usually operate with a spectral efficiency around $1 \mathrm{bit} / \mathrm{sec} / \mathrm{Hz}[6-8]$. The lower bound (36) obtained for channel (a) corroborates the results of the previous subsection related to channels with small spread factors, that is, over such channels we should be able to communicate at $80 \%$ of the theoretical rate obtained over AWGN channels.

UA-OFDM systems are not genie-aided and have to spend some resources to acquire CSI at reception, with the consequence of decreasing the data rate. Insights on how CSI impact the information rate can be obtained through the numerical analysis of the ratio $C^{L 1} / C^{\text {coh }}$, where $C^{\text {coh }}$ is defined as the achievable rate of UA-OFDM systems with perfect channel knowledge at reception. According to Appendix C, we have

$$
C^{\text {coh }}=\lim _{K \rightarrow \infty} \frac{1}{K T} \mathbb{E}_{\mathbf{H}}\left\{\log \operatorname{det}\left(\mathbf{I}+\frac{P T}{N} \mathbf{H H}^{\dagger} \operatorname{diag}\left(\mathbf{r}_{w}\right)^{-1}\right)\right\},
$$

which corresponds to the first term on the RHS of (36). Note that $C^{\text {coh }}$ is also an upperbound on $C$. (This can easily be shown by noticing that $I(\mathbf{y} ; \mathbf{x}) \leq I(\mathbf{y} ; \mathbf{x} \mid \mathbf{H})$.) As shown in Figure 5, the penalty induced by the absence of CSI is stronger for channels with larger Doppler spread (estimating $\mathbf{H}$ gets more difficult as the channel starts to fluctuate more rapidly) and can lead up to a $30 \%$ rate loss for a SNR of $20 \mathrm{~dB}$. In addition, $C^{L 1} / C^{\text {coh }}$ decreases with the SNR, which indicates that CSI acquisition may become a rate-limiting factor at high SNR. 


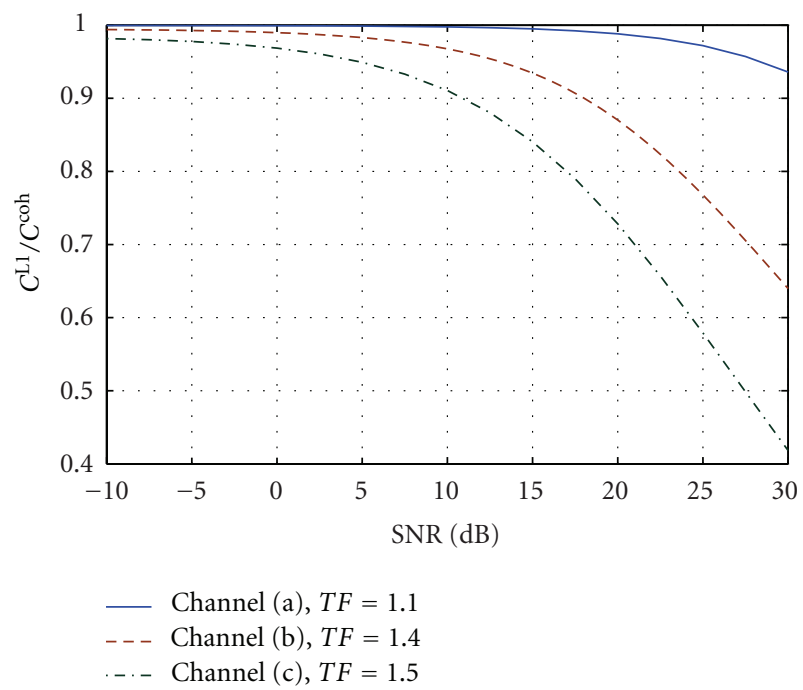

Figure 5: Lower-bound $C^{L 1}$ normalized with respect to coherent information rate $C^{\mathrm{coh}}$. For each channel, $T F$ is chosen such that there is no ISI.

\section{Conclusions and Perspectives}

The information-theoretic analysis provided in this paper led to the following conclusions:

(i) the information rate decreases with the channel spread factor but remains acceptable (i.e., greater than $1 \mathrm{bits} / \mathrm{sec} / \mathrm{Hz}$ ) as long as this factor is smaller than $10^{-1}$ and the signal-to-noise ratio is greater than $15 \mathrm{~dB}$;

(ii) numerical assessments on real UA channels with spread factors around $10^{-1}$ showed that reliable OFDM transmissions at 2 to $4 \mathrm{bits} / \mathrm{sec} / \mathrm{Hz}$ are achievable provided an average signal-to-noise ratio of 15 to $20 \mathrm{~dB}$;

(iii) current practices in designing OFDM systems for underwater channels are reasonable. More precisely, slightly oversizing guard intervals duration compared to the channel maximum delay spread is not much detrimental to the information rate, whereas underestimating this duration can be devastating.

Although quite realistic, the system model used in this paper could be more constrained. In particular, to strengthen our results, it would be interesting to add to our model a peak-power limitation, as in [31]. It is well known that OFDM systems can be sensitive to this limitation when power amplifiers do not operate with a large backoff. One way to tackle the problem would be to consider, in the information theoretic-treatment, the nonlinear distortion due to possible clipping as additional noise. Another point that deserves further attention is to study the information rate bound $C^{L 1}$ as a figure of merit for pulse-shaping optimization. While experimental results showed that large rates can be achieved with rectangular pulses, the bound provided in this paper could be tightened by maximizing it over all Weyl-Heisenberg sets.

\section{Appendices}

\section{A. Noise Model Approximation}

To model the ambient noise in the sea, four sources are usually considered: turbulence, shipping, waves, and thermal noise. These four noise components can be modeled by a colored Gaussian noise with the following empirical power spectral density (PSD) given in $\mathrm{dB}$ re $\mu \mathrm{Pa}^{2}$ per $\mathrm{Hz}$ as a function of frequency $f$ in $\mathrm{Hz}$ [24]:

$$
\begin{aligned}
& \text { turbulence: } 10 \log W_{t}(f)=17-30 \log \left(10^{-3} f\right), \\
& \text { shipping: } 10 \log W_{s}(f)=40+20(s-0.5)+26 \log \left(10^{-3} f\right) \\
& -60 \log \left(10^{-3} f+0.03\right), \\
& \text { waves: } 10 \log W_{w}(f)=50+7.5 v^{\frac{1}{2}}+20 \log \left(10^{-3} f\right) \\
& -40 \log \left(10^{-3} f+0.4\right),
\end{aligned}
$$$$
\text { thermal noise: } 10 \log W_{\mathrm{th}}(f)=-15+20 \log \left(10^{-3} f\right) \text {, }
$$

where $0 \leq s \leq 1$ is the shipping activity, and $v$ is the wind speed in $\mathrm{m} / \mathrm{s}$. The baseband equivalent noise PSD, as defined in $(8)$, is then given by

$$
\begin{aligned}
W(f)= & W_{t}\left(f+f_{c}\right)+W_{s}\left(f+f_{c}\right) \\
& +W_{w}\left(f+f_{c}\right)+W_{t h}\left(f+f_{c}\right),
\end{aligned}
$$

where $f_{c}$ is the carrier frequency corresponding the 0th subcarrier.

As noticed in [24], in the frequency region where most OFDM communication systems operate ( $1 \mathrm{kHz}$ to $100 \mathrm{kHz})$, the noise PSD decays almost linearly on the logarithmic scale. (Surface motion, caused by wind-driven waves is the main contributor to the noise in that frequency range.) This indicates that a simple expression may be found for the autocorrelation function $R_{w}(\tau)$. In fact, by expressing $R_{w}(\tau)$ as

$$
R_{w}(\tau)=\beta e^{-\mu|\tau|} e^{-j 2 \pi f_{c} \tau},
$$

where $\beta>0$ and $\mu>0$, we get the following PSD [32]:

$$
W(f)=\beta \frac{2 \mu}{\mu^{2}+4 \pi\left(f+f_{c}\right)^{2}},
$$

that turns out to be a good approximation of the noise PSD in frequency range of interest. This approximation is shown in Figure 6 with $\mu=5.10^{3}$, and $\beta$ is chosen such that the noise powers of models (A.2) and (A.4) perfectly match at $10 \mathrm{kHz}$. 


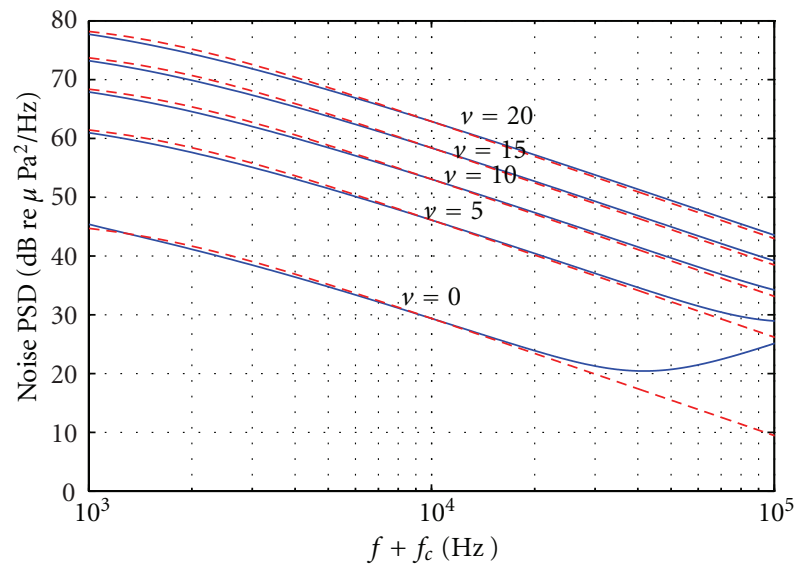

Figure 6: Power spectral density of the ambient noise for various wind speeds $v, s$ is set to 0.5 . The solid lines show the model (A.2) and dashed lines the one of (A.4). $f_{c}$ is the carrier frequency corresponding the 0 th subcarrier.

\section{B. Computation of the Signal and the Interference Power}

For all $k, k^{\prime}, n, n^{\prime} \in \mathbb{Z}$, we have that

$$
\begin{aligned}
& {\left[\mathbf{H}^{\left(k, k^{\prime}\right)}\right]_{n, n^{\prime}} \triangleq\left\langle\mathbb{U} g_{k^{\prime}, n^{\prime}}, \gamma_{k, n}\right\rangle} \\
& \stackrel{(\text { a) }}{=} \int_{t} \int_{\tau} \int_{\nu} G_{\mathbb{H}}(\tau, \nu) g\left(t-k^{\prime} T-\tau\right) e^{j 2 \pi n^{\prime} F(t-\tau)} \\
& \times e^{j 2 \pi \nu t} \gamma^{*}(t-k T) e^{-j 2 \pi n F t} d \nu d \tau d t \\
& \stackrel{(\mathrm{b})}{=} \int_{\tau} \int_{\nu} G_{\llbracket}(\tau, \nu) e^{-j 2 \pi n^{\prime} F \tau} \\
& \times\left[\int_{t} g^{*}\left(t-k^{\prime} T-\tau\right) \gamma(t-k T)\right. \\
& \left.\times e^{-j 2 \pi \nu t} e^{-j 2 \pi\left(n^{\prime}-n\right) F t} d t\right]^{*} d \nu d \tau \\
& \stackrel{(\mathrm{c})}{=} \int_{\tau} \int_{\nu} G_{\sharp}(\tau, \nu) \\
& \times A_{\gamma, g}^{*}\left(\tau+\left(k^{\prime}-k\right) T, \nu+\left(n^{\prime}-n\right) F\right) \\
& \times e^{-j 2 \pi n^{\prime} F \tau} e^{j 2 \pi\left(\nu+\left(n^{\prime}-n\right) F\right) k T} d \nu d \tau,
\end{aligned}
$$

where (a) and (b) follows from (9), (13), and (14), and (c) follows from the change of variables $t^{\prime}=t-k T$ and from (27).

From this expression, we can now derive the signal power

$$
\begin{aligned}
& \mathbb{E}\left\{\left|\left[\mathbf{H}^{(k, k)}\right]_{n, n}\right|^{2}\right\} \\
& \stackrel{(\text { a) }}{=} \mathbb{E}\left\{\mid \int_{\tau} \int_{\nu}\left(\bar{G}_{\mathbb{H}}(\tau, \nu)+\widetilde{G}_{\mathbb{H}}(\tau, \nu)\right)\right.
\end{aligned}
$$

$$
\begin{aligned}
& \left.\times\left. A_{\gamma, g}^{*}(\tau, \nu) e^{j 2 \pi(\nu k T-n F \tau)} d \nu d \tau\right|^{2}\right\}, \\
& \stackrel{(\mathrm{b})}{\approx} \mathbb{E}\left\{\left|\int_{\tau} \int_{\nu} \widetilde{G}_{\sharp}(\tau, v) A_{\gamma, g}^{*}(\tau, \nu) e^{j 2 \pi(v k T-n F \tau)} d \nu d \tau\right|^{2}\right\} \\
& +\left|\int_{\tau} \bar{h}_{\sharp}(\tau, k T) A_{\gamma, g}^{*}(\tau, 0) e^{-j 2 \pi n F \tau} d \tau\right|^{2}, \\
& \stackrel{(\mathrm{c})}{=} \int_{\tau} \int_{\nu} \int_{\tau^{\prime}} \int_{\nu^{\prime}} \mathbb{E}\left\{\widetilde{G}_{\mathbb{H}}(\tau, \nu) \widetilde{G}_{\mathbb{\sharp}}^{*}\left(\tau^{\prime}, \nu^{\prime}\right)\right\} A_{\gamma, g}^{*}(\tau, \nu) A_{\gamma, g}\left(\tau^{\prime}, \nu^{\prime}\right) \\
& \times e^{j 2 \pi(\nu k T-n F \tau)} \\
& \times e^{-j 2 \pi\left(\nu^{\prime} k T-n F \tau^{\prime}\right)} d \nu^{\prime} d \tau^{\prime} d \nu d \tau \\
& +\left|\int_{\tau} \bar{h}_{\llbracket}(\tau, k T) A_{\gamma, g}^{*}(\tau, 0) e^{-j 2 \pi n F \tau} d \tau\right|^{2}, \\
& \stackrel{(\mathrm{d})}{=} \int_{\tau} \int_{\tau^{\prime}} \int_{\nu} \tilde{S}_{\sharp}\left(\tau, \tau^{\prime}, \nu\right) A_{\gamma, g}^{*}(\tau, \nu) A_{\gamma, g}\left(\tau^{\prime}, \nu\right) \\
& \times e^{\mathrm{j} 2 \pi n F\left(\tau^{\prime}-\tau\right)} d \nu d \tau^{\prime} d \tau \\
& +\left|\int_{\tau} \bar{h}_{\mathbb{\sharp}}(\tau, k T) A_{\gamma, g}^{*}(\tau, 0) e^{-j 2 \pi n F \tau} d \tau\right|^{2},
\end{aligned}
$$

where (a) follows from (B.1) and (9). In (b), we use that $\mathbb{E}\left\{\widetilde{G}_{\sharp \mathbb{H}}(\tau, \nu)\right\}=0$, and we implicitly assume that the prototype functions $g(t)$ and $\gamma(t)$ have a compact support and that the channel average component $\bar{h}_{\sharp}(\tau, t)$ is approximately constant (in $t$ ) over that support. If we consider rectangular prototype functions, the duration of their support is upper-bounded by $T$, which represents a few tens or hundreds of milliseconds. This has to be compared with the fluctuation period of $\bar{h}_{\mathbb{H}}(\tau, t)$, which is rather a few tens or hundreds of seconds [2]. Note that, theoretically, some prototype functions can have an infinite support. However, the contribution to the received power from the part of the pulse that has a support greater than few times the symbol period $T$ is rather negligible (e.g., for most infinitelength prototype functions such as Gaussian, Raised-Cosine, etc., we usually have that $\left.\int_{-5 T}^{5 T}|g(t)|^{2} d t \approx \int_{-\infty}^{\infty}|g(t)|^{2} d t\right)$. (d) holds because, according to (4), the zero-mean random

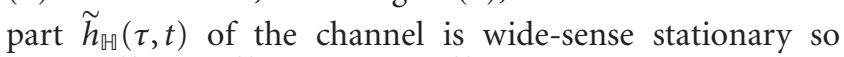

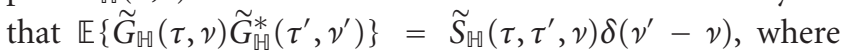
$\tilde{S}_{\mathbb{H}}\left(\tau, \tau^{\prime}, \nu\right)$ is the channel scattering function defined in (29). Note that in the case where the scatterers are assumed to be uncorrelated $\mathbb{E}\left\{\widetilde{G}_{\mathbb{\sharp}}(\tau, \nu) \widetilde{G}_{\varpi \mathbb{t}}^{*}\left(\tau^{\prime}, \nu^{\prime}\right)\right\}=\widetilde{S}_{\mathbb{H}}(\tau, \nu) \delta\left(\nu^{\prime}-\nu\right) \delta\left(\tau^{\prime}-\right.$ $\tau$ ), so that (B.2) simplifies to

$$
\begin{aligned}
& \mathbb{E}\left\{\left|\left[\mathbf{H}^{(k, k)}\right]_{n, n}\right|^{2}\right\} \\
& \approx \int_{\tau} \int_{\nu} \widetilde{S}_{\mathbb{\sharp}}(\tau, \nu)\left|A_{\gamma, g}(\tau, \nu)\right|^{2} d \nu d \tau \\
& \quad+\left|\int_{\tau} \bar{h}_{\mathbb{\sharp}}(\tau, k T) A_{\gamma, g}^{*}(\tau, 0) e^{-j 2 \pi n F \tau} d \tau\right|^{2} .
\end{aligned}
$$


Similarly to (B.2), the interference power can be derived from the following development:

$$
\begin{aligned}
& \mathbb{E}\left\{\left|\left[\mathbf{H}^{\left(k, k^{\prime}\right)}\right]_{n, n^{\prime}}\right|^{2}\right\} \\
& \stackrel{(\mathrm{a})}{=} \mathbb{E}\left\{\mid \int_{\tau} \int_{\nu}\left(\bar{G}_{\mathbb{W}}(\tau, \nu)+\widetilde{G}_{\mathbb{W}}(\tau, \nu)\right)\right. \\
& \times A_{\gamma, g}^{*}\left(\tau+\left(k^{\prime}-k\right) T, \nu+\left(n^{\prime}-n\right) F\right) \\
& \left.\times\left. e^{-j 2 \pi n^{\prime} F \tau} e^{j 2 \pi\left(\nu+\left(n^{\prime}-n\right) F\right) k T} d \nu d \tau\right|^{2}\right\} \\
& \stackrel{(\mathrm{b})}{\approx} \mathbb{E}\left\{\mid \int_{\tau} \int_{\nu} \widetilde{G}_{\mathbb{H}}(\tau, \nu) A_{\gamma, g}^{*}\left(\tau+\left(k^{\prime}-k\right) T, \nu+\left(n^{\prime}-n\right) F\right)\right. \\
& \left.\times\left. e^{-j 2 \pi n^{\prime} F \tau} e^{j 2 \pi\left(\nu+\left(n^{\prime}-n\right) F\right) k T} d \nu d \tau\right|^{2}\right\} \\
& +\mid \int_{\tau} \bar{h}_{\llbracket(}\left(\tau, k^{\prime} T\right) \\
& \times\left. A_{\gamma, g}^{*}\left(\tau+\left(k^{\prime}-k\right) T,\left(n^{\prime}-n\right) F\right) e^{-j 2 \pi n^{\prime} F \tau} d \tau\right|^{2} \\
& \stackrel{(\mathfrak{c})}{=} \int_{\tau} \int_{\tau^{\prime}} \int_{\nu} \widetilde{S}_{\mathfrak{U}}\left(\tau, \tau^{\prime}, \nu\right) \\
& \times A_{\gamma, g}^{*}\left(\tau+\left(k^{\prime}-k\right) T, \nu+\left(n^{\prime}-n\right) F\right) \\
& \times A_{\gamma, g}\left(\tau^{\prime}+\left(k^{\prime}-k\right) T, \nu+\left(n^{\prime}-n\right) F\right) \\
& \times e^{j 2 \pi n^{\prime} F\left(\tau^{\prime}-\tau\right)} d \nu d \tau^{\prime} d \tau \\
& +\mid \int_{\tau} \bar{h}_{\mathbb{\square}}\left(\tau, k^{\prime} T\right) \\
& \times\left. A_{\gamma, g}^{*}\left(\tau+\left(k^{\prime}-k\right) T,\left(n^{\prime}-n\right) F\right) e^{-j 2 \pi n^{\prime} F \tau} d \tau\right|^{2},
\end{aligned}
$$

where (a) follows from (B.1) and (9). For (B.2) and (B.4) alike, in (b) we assume that $\bar{h}_{\mathbb{\square}}(\tau, t)$ is approximately constant over some period of time. We here consider that $\bar{h}_{\mathbb{U}}(\tau, t)$ does not fluctuate much over the duration that corresponds to the maximal time difference between two interfering OFDM symbols, that is, $\bar{h}_{\mathbb{\square}}(\tau, k T) \approx$ $\bar{h}_{\square \square}\left(\tau, k^{\prime} T\right), \forall\left(k, k^{\prime}\right) \in\left\{\left(k, k^{\prime}\right) \mid \mathbb{E}\left\{\left|\left[\mathbf{H}^{\left(k, k^{\prime}\right)}\right]_{n, n^{\prime}}\right|^{2}\right\} \neq 0\right\}$. Once again, this assumption is not restricting since for most OFDM systems the duration $\left(k^{\prime}-k\right) T$ only represents a few tens or hundreds of milliseconds in worst-case scenarios. In the case where the scatterers are assumed to be uncorrelated, (B.4) simplifies to

$$
\begin{aligned}
& \mathbb{E}\left\{\left|\left[\mathbf{H}^{\left(k, k^{\prime}\right)}\right]_{n, n^{\prime}}\right|^{2}\right\} \\
& =\int_{\tau} \int_{\nu} \tilde{S}_{\mathbb{t}-\mathbb{t}}(\tau, \nu) \\
& \quad \times\left|A_{\gamma, g}\left(\tau+\left(k^{\prime}-k\right) T, \nu+\left(n^{\prime}-n\right) F\right)\right|^{2} d \nu d \tau
\end{aligned}
$$

$$
\begin{aligned}
& +\mid \int_{\tau} \bar{h}_{\mathbb{T}}\left(\tau, k^{\prime} T\right) \\
& \quad \times\left. A_{\gamma, g}^{*}\left(\tau+\left(k^{\prime}-k\right) T,\left(n^{\prime}-n\right) F\right) e^{-j 2 \pi n^{\prime} F \tau} d \tau\right|^{2} .
\end{aligned}
$$

\section{Proof of Theorem 1}

A lower bound on $C$ can be obtained by evaluating the mutual information $I(\mathbf{y} ; \mathbf{x})$ for a specific input distribution. Specifically, $\mathbf{x}$ is chosen such that $\mathbf{x} \sim \mathcal{C} \mathcal{N}(0,(P T / N) \mathbf{I})$. The proof of Theorem 1 next relies on the following information theoretic inequality $[29,33]$ :

$$
I(\mathbf{y} ; \mathbf{x}) \geq I(\mathbf{y} ; \mathbf{x} \mid \mathbf{H})-I(\mathbf{y} ; \mathbf{H} \mid \mathbf{x}) .
$$

C.1. Computation of $I(y ; x \mid H)$. The computation of $I(\mathbf{y} ; \mathbf{x} \mid$ $\mathbf{H})=h_{E}(\mathbf{y} \mid \mathbf{H})-h_{E}(\mathbf{y} \mid \mathbf{x}, \mathbf{H})$ is straightforward since

(i) conditional on $\mathbf{H}, \mathbf{y}$ is distributed according to a complex Gaussian distribution with a covariance matrix equal to $(P T / N) \mathbf{H H}^{\dagger}+\mathbb{E}\left\{\mathbf{w} \mathbf{w}^{\dagger}\right\}$;

(ii) conditional on $\mathbf{x}$ and $\mathbf{H}, \mathbf{y}$ is complex Gaussian with a covariance matrix equal to $\mathbb{E}\left\{\mathbf{w} \mathbf{w}^{\dagger}\right\}$.

The entries of the $N K \times N K$ noise covariance matrix $\mathbb{E}\left\{\mathbf{w} \mathbf{w}^{\dagger}\right\}$ are given by

$$
\begin{aligned}
& {\left[\mathbb{E}\left\{\mathbf{w} \mathbf{w}^{\dagger}\right\}^{\left(k, k^{\prime}\right)}\right]_{n, n^{\prime}} \triangleq \mathbb{E} }\left\{w_{k, n} w_{k^{\prime}, n^{\prime}}^{*}\right\} \\
&= \int_{\tau} R_{w}(\tau) e^{-j 2 \pi n F \tau} \\
& \times A_{\gamma, \gamma}^{*}\left(\tau+\left(k^{\prime}-k\right) T,\left(n^{\prime}-n\right) F\right) \\
& \times e^{j 2 \pi k T F\left(n^{\prime}-n\right)} d \tau \\
& \stackrel{(\mathrm{a})}{\approx} \int_{\tau} R_{w}(\tau) e^{-j 2 \pi n F \tau} \\
& \times A_{\gamma, \gamma}^{*}\left(\left(k^{\prime}-k\right) T,\left(n^{\prime}-n\right) F\right) \\
& \times e^{j 2 \pi k T F\left(n^{\prime}-n\right)} d \tau \\
& \stackrel{(\mathrm{b})}{=} \int_{\tau} R_{w}(\tau) e^{-j 2 \pi n F \tau} d \tau \\
& \times \delta\left(k-k^{\prime}\right) \delta\left(n-n^{\prime}\right) \\
& \stackrel{(\mathrm{c})}{=} W(n F) \times \delta\left(k-k^{\prime}\right) \delta\left(n-n^{\prime}\right) .
\end{aligned}
$$

Here, (a) is based on Appendix A where it is shown that $R_{w}(\tau)$ can be well approximated by a function that decays 
very fast compared to common pulse durations (i.e., $R_{w}(\tau)=$ $\beta e^{-5.10^{3}|\tau|} e^{-j 2 \pi f_{c} \tau}$ ). (b) follows from the orthogonality of the receive pulse and (c) from (8). Consequently,

$$
\begin{aligned}
I(\mathbf{y} ; \mathbf{x} \mid \mathbf{H}) & =\mathbb{E}_{\mathbf{H}}\left\{\log \operatorname{det}\left(\mathbf{I}+\frac{P T}{N} \mathbf{H H}^{\dagger} \mathbb{E}\left\{\mathbf{w w}^{\dagger}\right\}^{-1}\right)\right\} \\
& =\mathbb{E}_{\mathbf{H}}\left\{\log \operatorname{det}\left(\mathbf{I}+\frac{P T}{N} \mathbf{H H}^{\dagger} \operatorname{diag}\left(\mathbf{r}_{w}\right)^{-1}\right)\right\},
\end{aligned}
$$

where the entries of the $N K \times 1$ vector $\mathbf{r}_{w}$ are defined as

$$
r_{w}(n+k K) \triangleq W(n F), \quad n \in[0, N-1], \quad k \in[0, K-1] .
$$

C.2. Computation of $I(y ; H \mid x)$. The offdiagonal elements of $\mathbf{H}$ being generally nonnull in highly dispersive environments, the derivation of $I(\mathbf{y} ; \mathbf{H} \mid \mathbf{x})$ is not that easy. Influenced by [21], we next seek an upper bound on the penalty term $I(\mathbf{y} ; \mathbf{H x})$ by splitting $\mathbf{y}$ into an interference-free part and an interference-only part, so that

$$
\begin{aligned}
\mathbf{y} & =\mathbf{H} \mathbf{x}+\mathbf{w} \\
& =\mathbf{h} \odot \mathbf{x}+\mathbf{Z x}+\mathbf{x} \\
& =\underbrace{\mathbf{h} \odot \mathbf{x}+\mathbf{w}_{1}}_{\triangleq \mathbf{y}_{1}}+\underbrace{\mathbf{Z x}+\mathbf{w}_{2}}_{\triangleq \mathbf{y}_{2}},
\end{aligned}
$$

where $\mathbf{w}_{1}$ are two independent random vectors such that $\mathbf{w}_{1} \sim \mathcal{C} \mathcal{N}\left(0, \alpha \times \operatorname{diag}\left(\mathbf{r}_{w}\right)\right)$ and $\mathbf{w}_{2} \sim \mathcal{C} \mathcal{N}(0,(1-\alpha) \times$ $\left.\operatorname{diag}\left(\mathbf{r}_{w}\right)\right)$, with $0<\alpha<1$.

Let us note that

$$
\begin{aligned}
& I(\mathbf{y} ; \mathbf{H} \mid \mathbf{x}) \stackrel{(a)}{\leq} I\left(\mathbf{y}_{1}, \mathbf{y}_{2} ; \mathbf{H} \mid \mathbf{x}\right) \\
& \stackrel{\text { (b) }}{\leq} I\left(\mathbf{y}_{1}, \mathbf{y}_{2} ; \mathbf{h}, \mathbf{Z} \mid \mathbf{x}\right) \\
& \stackrel{(c)}{=} I\left(\mathbf{y}_{1} ; \mathbf{h}, \mathbf{Z} \mid \mathbf{x}\right)+I\left(\mathbf{y}_{2} ; \mathbf{h}, \mathbf{Z} \mid \mathbf{x}, \mathbf{y}_{1}\right) \\
& \stackrel{(\mathrm{d})}{=} I\left(\mathbf{y}_{1} ; \mathbf{h} \mid \mathbf{x}\right)+I\left(\mathbf{y}_{2} ; \mathbf{h}, \mathbf{Z} \mid \mathbf{x}, \mathbf{y}_{1}\right) \\
& \stackrel{(e)}{=} I\left(\mathbf{y}_{1} ; \mathbf{h} \mid \mathbf{x}\right)+h_{E}\left(\mathbf{y}_{2} \mid \mathbf{x}, \mathbf{y}_{1}\right) \\
& -h_{E}\left(\mathbf{y}_{2} \mid \mathbf{x}, \mathbf{y}_{1}, \mathbf{h}, \mathbf{Z}\right) \\
& \stackrel{(\mathrm{f})}{=} I\left(\mathbf{y}_{1} ; \mathbf{h} \mid \mathbf{x}\right)+h_{E}\left(\mathbf{y}_{2} \mid \mathbf{x}, \mathbf{y}_{1}\right) \\
& -h_{E}\left(\mathbf{y}_{2} \mid \mathbf{x}, \mathbf{Z}\right) \\
& \stackrel{(\mathrm{g})}{\leq} I\left(\mathbf{y}_{1} ; \mathbf{h} \mid \mathbf{x}\right)+h_{E}\left(\mathbf{y}_{2} \mid \mathbf{x}\right) \\
& -h_{E}\left(\mathbf{y}_{2} \mid \mathbf{x}, \mathbf{Z}\right) \\
& =I\left(\mathbf{y}_{1} ; \mathbf{h} \mid \mathbf{x}\right)+I\left(\mathbf{y}_{2} ; \mathbf{Z} \mid \mathbf{x}\right) \text {. }
\end{aligned}
$$

In (a) and (b), we used the data processing inequality, (c) follows from the chain rule, (d) holds because $\mathbf{y}_{1}$ and $\mathbf{Z}$ are conditionally independent given $\mathbf{h}$, in (e) we expressed mutual information as a function of entropy, (f) holds because $\mathbf{y}_{2}$ and $\mathbf{y}_{1}$ are conditionally independent given $\mathbf{x}$ and $\mathbf{h}$, and also because $\mathbf{y}_{2}$ and $\mathbf{h}$ are conditionally independent given $\mathbf{x}$ and $\mathbf{Z}$. Finally, the fact that conditioning reduces entropy leads to $(\mathrm{g})$.

Using that $\mathbf{y}_{1}$ is Gaussian given $\mathbf{h}$ and $\mathbf{x}$, and as a consequence of Jensen's inequality, $I\left(\mathbf{y}_{1} ; \mathbf{h} \mid \mathbf{x}\right)$ can be upperbounded as

$$
\begin{aligned}
& I\left(\mathbf{y}_{1} ; \mathbf{h} \mid \mathbf{x}\right) \\
& =\mathbb{E}_{x}\left\{\log \operatorname{det}\left(\mathbf{I}+\frac{\operatorname{diag}(\mathbf{x}) \operatorname{diag}\left(\mathbf{x}^{\dagger}\right)}{\alpha} \mathbf{R}_{\mathbf{h}} \operatorname{diag}\left(\mathbf{r}_{w}\right)^{-1}\right)\right\} \\
& \leq \log \operatorname{det}\left(\mathbf{I}+\frac{P T}{N \alpha} \mathbf{R}_{\mathbf{h}} \operatorname{diag}\left(\mathbf{r}_{w}\right)^{-1}\right),
\end{aligned}
$$

where $\mathbf{R}_{\mathbf{h}}$ denotes the covariance matrix of the direct channel vector $\mathbf{h}$. From (B.1), we can express the entries of $\mathbf{R}_{\mathbf{h}}$ as

$$
\begin{aligned}
{\left[\mathbf{R}_{\mathrm{h}}^{\left(k, k^{\prime}\right)}\right]_{n, n^{\prime}}=\int_{\tau} \int_{\tau^{\prime}} \int_{\nu} \tilde{S}_{\boxplus \Perp} } & \left(\tau, \tau^{\prime}, \nu\right) A_{\gamma, g}^{*}(\tau, \nu) \\
& \times A_{\gamma, g}\left(\tau^{\prime}, \nu\right) e^{j 2 \pi \nu T\left(k-k^{\prime}\right)} \\
& \times e^{j 2 \pi F\left(n^{\prime} \tau^{\prime}-n \tau\right)} d \nu d \tau^{\prime} d \tau .
\end{aligned}
$$

We next seek an upper bound on $I\left(\mathbf{y}_{2} ; \mathbf{Z} \mid \mathbf{x}\right)$. Let $\mathbf{Q}(\mathbf{x})=\mathbb{E}_{\mathbf{Z}}\left\{\left(\mathbf{Z} \mathbf{x}-\mathbb{E}_{\mathbf{Z}}\{\mathbf{Z} \mathbf{x}\}\right)\left(\mathbf{Z} \mathbf{x}-\mathbb{E}_{\mathbf{Z}}\{\mathbf{Z} \mathbf{x}\}\right)^{\dagger}\right\}$ be the conditional covariance matrix of the vector $\mathbf{Z x}$ given $\mathbf{x}$. $\mathbf{Z x}$ being Gaussian given $\mathbf{x}$, using Hadamard's and Jensen's inequalities, $I\left(\mathbf{y}_{2} ; \mathbf{Z} \mid \mathbf{x}\right)$ is then upper-bounded as follows:

$$
\begin{aligned}
& I\left(\mathbf{y}_{2} ; \mathbf{Z} \mid \mathbf{x}\right) \\
& \quad=\mathbb{E}_{x}\left\{\log \operatorname{det}\left(\mathbf{I}+\frac{1}{1-\alpha} \mathbf{Q}(\mathbf{x}) \operatorname{diag}\left(\mathbf{r}_{w}\right)^{-1}\right)\right\} \\
& \quad \leq \sum_{k=0}^{K-1} \sum_{n=0}^{N-1} \mathbb{E}_{x}\left\{\log \left(1+\frac{1}{(1-\alpha) r_{w}(n+k K)}\left[\mathbf{Q}(\mathbf{x})^{(k, k)}\right]_{n, n}\right)\right\} \\
& \leq \sum_{k=0}^{K-1} \sum_{n=0}^{N-1} \log \left(1+\frac{1}{(1-\alpha) r_{w}(n+k K)} \mathbb{E}_{x}\left\{\left[\mathbf{Q}(\mathbf{x})^{(k, k)}\right]_{n, n}\right\}\right) \\
& =\sum_{k=0}^{K-1} \sum_{n=0}^{N-1} \log \left(1+\frac{P T}{N(1-\alpha) r_{w}(n+k K)} \widetilde{\sigma}_{I_{n}}^{2}\right),
\end{aligned}
$$

where the last equality holds because the input symbols are i.i.d. with zero mean, so that

$$
\begin{aligned}
& \mathbb{E}_{x}\left\{\left[\mathbf{Q}(\mathbf{x})^{(k, k)}\right]_{n, n}\right\} \\
& \quad=\frac{P T}{N}\left[\mathbb{E}\left\{(\mathbf{Z}-\mathbb{E}\{\mathbf{Z}\})(\mathbf{Z}-\mathbb{E}\{\mathbf{Z}\})^{\dagger}\right\}^{(k, k)}\right]_{n, n} \\
& \quad=\frac{P T}{N} \widetilde{\sigma}_{I_{n}}^{2},
\end{aligned}
$$

with $\widetilde{\sigma}_{I_{n}}^{2}$ the interference power due to the random part of the channel as defined in (31). Equation (C.9) can be further 
simplified by noticing that $r_{w}(n+k K)$ and $\tilde{\sigma}_{I_{n}}^{2}$ do not depend on $k$; therefore,

$$
I\left(\mathbf{y}_{2} ; \mathbf{Z} \mid \mathbf{x}\right) \leq K \sum_{n=0}^{N-1} \log \left(1+\frac{P T}{N(1-\alpha) r_{w}(n)} \widetilde{\sigma}_{I_{n}}^{2}\right) .
$$

From (C.1), (C.3), (C.7), and (C.11), and for all $0<\alpha<$ $1, I(\mathbf{y} ; \mathbf{x})$ can be lower-bounded as follows:

$$
\begin{aligned}
I(\mathbf{y} ; \mathbf{x}) \geq & \mathbb{E}_{\mathbf{H}}\left\{\log \operatorname{det}\left(\mathbf{I}+\frac{P T}{N} \mathbf{H H}^{\dagger} \operatorname{diag}\left(\mathbf{r}_{w}\right)^{-1}\right)\right\} \\
& -\left[\log \operatorname{det}\left(\mathbf{I}+\frac{P T}{N \alpha} \mathbf{R}_{\mathbf{h}} \operatorname{diag}\left(\mathbf{r}_{w}\right)^{-1}\right)\right. \\
& \left.+K \sum_{n=0}^{N-1} \log \left(1+\frac{P T}{N(1-\alpha) r_{w}(n)} \widetilde{\sigma}_{I_{n}}^{2}\right)\right] .
\end{aligned}
$$

The bound is then tightened by choosing $\alpha$ that minimizes the penalty term, which concludes the proof.

\section{Proof of Corollary 2}

D.1. White Noise Assumption. Under the white noise assumption, the noise PSD is flat over the entire bandwidth so that $W(f)=W_{0}$, where $W_{0}$ is a constant. In that case, the entries of vector $\mathbf{r}_{w}$ are all equal to $W_{0}$, and the SNR $\rho$ satisfies $\rho=P /\left(B W_{0}\right)$. Given that $B=N F$, from Theorem 1 we have

$$
\begin{aligned}
C \geq & \lim _{K \rightarrow \infty} \frac{1}{K T} \mathbb{E}_{\mathbf{H}}\left\{\log \operatorname{det}\left(\mathbf{I}+\rho T F \mathbf{H H}^{\dagger}\right)\right\} \\
& -\inf _{0<\alpha<1} \frac{1}{K T}\left[\log \operatorname{det}\left(\mathbf{I}+\frac{\rho T F}{\alpha} \mathbf{R}_{\mathbf{h}}\right)\right. \\
& \left.+K \sum_{n=0}^{N-1} \log \left(1+\frac{\rho T F}{1-\alpha} \widetilde{\sigma}_{I_{n}}^{2}\right)\right] .
\end{aligned}
$$

D.2. Uncorrelated Scattering Assumption. In the case where the scatterers can be assumed as uncorrelated, the lower bound on the information rate can be further simplified.

First, the channel scattering function is reduced from three to two dimensions so that $\tilde{\sigma}_{I_{n}}^{2}$ does not depend on $n$ anymore and is expressed as

$$
\begin{aligned}
\tilde{\sigma}_{I_{n}}^{2}=\sum_{\substack{k^{\prime}=1-K n^{\prime}=1-N \\
\left(k^{\prime}, n^{\prime}\right) \neq(0,0)}}^{K-1} \int_{\tau} \int_{\nu} \tilde{S}_{\bullet 甘}(\tau, \nu) \\
\\
\quad \times\left|A_{\gamma, g}^{*}\left(\tau+k^{\prime} T, \nu+n^{\prime} F\right)\right|^{2} d \nu d \tau
\end{aligned}
$$$$
\triangleq \widetilde{\sigma}_{I}^{2}
$$

Second, the covariance matrix $\mathbf{R}_{\mathbf{h}}$ becoming block-Toeplitz, the extension of Szegö's theorem to two-level Toeplitz matrices can be applied, that is [34, Th. 3]:

$$
\begin{aligned}
& \lim _{K \rightarrow \infty} \frac{1}{K T} \log \operatorname{det}\left(\mathbf{I}+\frac{\rho T F}{\alpha} \mathbf{R}_{\mathbf{h}}\right) \\
&=\frac{1}{T} \int_{-1 / 2}^{1 / 2} \log \operatorname{det}\left(\mathbf{I}+\frac{\rho T F}{\alpha} \widetilde{\mathbf{S}}(\theta)\right) d \theta,
\end{aligned}
$$

where $\widetilde{\mathbf{S}}(\theta)$ is the power spectral density of the zero-mean multivariate random process $\left\{\mathbf{h}_{k}-\mathbb{E}\left\{\mathbf{h}_{k}\right\}\right\}$, with $\mathbf{h}_{k} \triangleq$ $\left[h_{k, 0} h_{k, 1} \cdots h_{k, N-1}\right]^{T}$, that is,

$$
\widetilde{\mathbf{S}}(\theta) \triangleq \sum_{k=-\infty}^{\infty} \mathbb{E}\left\{\left(\mathbf{h}_{k}-\mathbb{E}\left\{\mathbf{h}_{k}\right\}\right)\left(\mathbf{h}_{k}-\mathbb{E}\left\{\mathbf{h}_{k}\right\}\right)^{\dagger}\right\} e^{-j 2 \pi k \theta} .
$$

By noticing that the entries on the main diagonal of $\widetilde{\mathbf{S}}(\theta)$ are all equal and by applying Hadamard's inequality, we have

$$
\begin{aligned}
& \frac{1}{T} \int_{-1 / 2}^{1 / 2} \log \operatorname{det}\left(\mathbf{I}+\frac{\rho T F}{\alpha} \widetilde{\mathbf{S}}(\theta)\right) d \theta \\
& \quad \leq \frac{N}{T} \int_{-1 / 2}^{1 / 2} \log \left(1+\frac{\rho T F}{\alpha} \widetilde{s}(\theta)\right) d \theta,
\end{aligned}
$$

where $\widetilde{s}(\theta)$ is the PSD of the zero-mean stationary channel process $\left\{h_{k, n}-\mathbb{E}\left\{h_{k, n}\right\}\right\}_{k}$ and is expressed as

$$
\widetilde{\mathcal{S}}(\theta)=\sum_{k=-\infty}^{\infty} \int_{\tau} \int_{\nu} \tilde{S}_{\mathbb{H}}(\tau, \nu)\left|A_{\gamma, g}(\tau, \nu)\right|^{2} e^{j 2 \pi \nu k T} d \nu d \tau e^{-j 2 \pi k \theta} .
$$

Corollary 2 is then obtained by noticing that $N / T=B /(T F)$.

\section{Acknowledgments}

The authors are grateful to the DGA-TN for providing part of the experimental data used in this paper. This work was supported in part by the ONR MURI Grant N00014-07-10738, the ONR Grant N00014-10-1-0576, and the Grant NSF 0831728.

\section{References}

[1] P. van Walree, "Channel sounding for acoustic communications: techniques and shallow-water examples," Research Report, Norwegian Defence Research Establishment (FFI), Kjeller, Norway, 2011.

[2] F.-X. Socheleau, C. Laot, and J.-M. Passerieux, "Stochastic replay of non-WSSUS underwater acoustic communication channels recorded at sea," IEEE Transactions on Signal Processing, vol. 59, no. 10, pp. 4838-4849, 2011.

[3] M. Stojanovic and J. Preisig, "Underwater acoustic communication channels: propagation models and statistical characterization," IEEE Communications Magazine, vol. 47, no. 1, pp. 84-89, 2009.

[4] B. Tomasi, J. Preisig, G. B. Deane, and M. Zorzi, "A study on the wide-sense stationarity of the underwater acoustic channel for non-coherent communication systems," in Proceedings of the 11th European Wireless Conference, Sustainable Wireless Technologies (European Wireless), pp. 1-6, April 2011. 
[5] D. B. Kilfoyle and A. B. Baggeroer, "State of the art in underwater acoustic telemetry," IEEE Journal of Oceanic Engineering, vol. 25, no. 1, pp. 4-27, 2000.

[6] F. Frassati, C. Lafon, P. A. Laurent, and J. M. Passerieux, "Experimental assessment of OFDM and DSSS modulations for use in littoral waters underwater acoustic communications," in Proceedings of IEEE Oceans'05 Europe Conference \& Exhibition, pp. 826-831, Brest, France, June 2005.

[7] B. Li, S. Zhou, M. Stojanovic, L. L. Freitag, and P. Willett, "Multicarrier communication over underwater acoustic channels with nonuniform Doppler shifts," IEEE Journal of Oceanic Engineering, vol. 33, no. 2, pp. 198-209, 2008.

[8] C. R. Berger, S. Zhou, J. C. Preisig, and P. Willett, "Sparse channel estimation for multicarrier underwater acoustic communication: from subspace methods to compressed sensing," IEEE Transactions on Signal Processing, vol. 58, no. 3, pp. 1708$1721,2010$.

[9] G. Leus and P. A. Van Walree, "Multiband OFDM for covert acoustic communications," IEEE Journal on Selected Areas in Communications, vol. 26, no. 9, Article ID 4686805, pp. 1662 1673, 2008.

[10] P. Jung and G. Wunder, "The WSSUS pulse design problem in multicarrier transmission," IEEE Transactions on Communications, vol. 55, no. 10, pp. 1918-1928, 2007.

[11] G. Matz, D. Schafhuber, K. Gröchenig, M. Hartmann, and F. Hlawatsch, "Analysis, optimization, and implementation of low-interference wireless multicarrier systems," IEEE Transactions on Wireless Communications, vol. 6, no. 5, pp. 1921-1930, 2007.

[12] A. Barbieri, G. Caire, and U. Mitra, "Transmit/receive filter optimization for doubly-selective underwater acoustic channels," in Proceedings of the IEEE OCEANS' 2008, pp. 1-6, September 2008.

[13] W. Kozek and A. F. Molisch, "Nonorthogonal pulseshapes for multicarrier communications in doubly dispersive channels," IEEE Journal on Selected Areas in Communications, vol. 16, no. 8, pp. 1579-1589, 1998.

[14] R. Haas and J. C. Belfiore, "A Time-Frequency Well-localized Pulse for Multiple Carrier Transmission," Wireless Personal Communications, vol. 5, no. 1, pp. 1-18, 1997.

[15] K. Liu, T. Kadous, and A. M. Sayeed, "Orthogonal timefrequency signaling over doubly dispersive channels," IEEE Transactions on Information Theory, vol. 50, no. 11, pp. 25832603, 2004.

[16] P. A. Bello, "Characterization of randomly time-variant linear channels," IEEE Transactions on Communications Systems, vol. 11, no. 4, pp. 360-393, 1963.

[17] C. Polprasert, J. A. Ritcey, and M. Stojanovic, "Capacity of OFDM systems over fading underwater acoustic channels," IEEE Journal of Oceanic Engineering, vol. 36, no. 4, pp. 514 524, 2011.

[18] M. C. Gursoy, H. V. Poor, and S. Verdú, "The noncoherent Rician fading channel part I: structure of the capacityachieving input," IEEE Transactions on Wireless Communications, vol. 4, no. 5, pp. 2193-2206, 2005.

[19] V. Sethuraman, L. Wang, B. Hajek, and A. Lapidoth, "LowSNR capacity of noncoherent fading channels," IEEE Transactions on Information Theory, vol. 55, no. 4, pp. 1555-1574, 2009.

[20] G. Durisi, U. G. Schuster, H. Bölcskei, and S. Shamai, "Noncoherent capacity of underspread fading channels," IEEE Transactions on Information Theory, vol. 56, no. 1, Article ID 5361496, pp. 367-395, 2010.
[21] G. Durisi, V. I. Morgenshtern, and H. Bolcskei, "Sensitivity of continuous-time noncoherent fading channel capacity," IEEE Transactions on Information Theory. In press.

[22] P. Qarabaqi and M. Stojanovic, "Statistical modeling of a shallow water acoustic communication channel," in Proceedings of the Underwater Acoustic Measurements: Technologies and Results Conference, p. 13411350, Nafplion, Greece, June 2009.

[23] F. D. Neeser and J. L. Massey, "Proper complex random processes with applications to information theory," IEEE Transactions on Information Theory, vol. 39, no. 4, pp. 1293 1303, 1993.

[24] M. Stojanovic, "On the relationship between capacity and distance in an underwater acoustic communication channel," ACM SIGMOBILE Mobile Computing and Communications Review (MC2R), vol. 11, no. 4, pp. 43-64, 2007.

[25] O. Christensen, An Introduction to Frames and Riesz Bases, Birkhuser, Boston, Mass, USA, 2003.

[26] F. Hlawatsch and G. Matz, Wireless Communications Over Rapidly Time-Varying Channels, Elsevier, New York, NY, USA, 2011.

[27] S.-J. Hwang and P. Schniter, "Efficient multicarrier communication for highly spread underwater acoustic channels," IEEE Journal on Selected Areas in Communications, vol. 26, no. 9, Article ID 4686806, pp. 1674-1683, 2008.

[28] D. Schafhuber, H. Bölcskei, and G. Matz, "System capacity of wideband OFDM communications over fading channels without channel knowledge," in Proceedings of the IEEE International Symposium on Information Theory (ISIT '04), July 2004.

[29] X. Deng and A. M. Haimovich, "Achievable rates over time-varying rayleigh fading channels," IEEE Transactions on Communications, vol. 55, no. 7, pp. 1397-1406, 2007.

[30] W. Kozek, Matched weyl-heisenberg expansions of nonstationary environments [Ph.D. thesis], Department of Electrical Engineering, Vienna University of Technology, Vienna, Austria, 1997.

[31] J.-M. Passerieux, F.-X. Socheleau, and C. Laot, "On the capacity of the underwater acoustic communication channel under realistic assumptions," in Proceedings of the IEEE European Wireless, pp. 1-6, April 2011.

[32] G. Campbell and R. Foster, Fourier Integrals for Practical Applications, D Van Nostrand Company Inc, New York, NY, USA, 1948.

[33] V. Sethuraman and B. Hajek, "Capacity per unit energy of fading channels with a peak constraint," IEEE Transactions on Information Theory, vol. 51, no. 9, pp. 3102-3120, 2005.

[34] H. Gazzah, P. A. Regalia, and J. P. Delmas, "Asymptotic eigenvalue distribution of block toeplitz matrices and application to blind SIMO channel identification," IEEE Transactions on Information Theory, vol. 47, no. 3, pp. 1243-1251, 2001. 

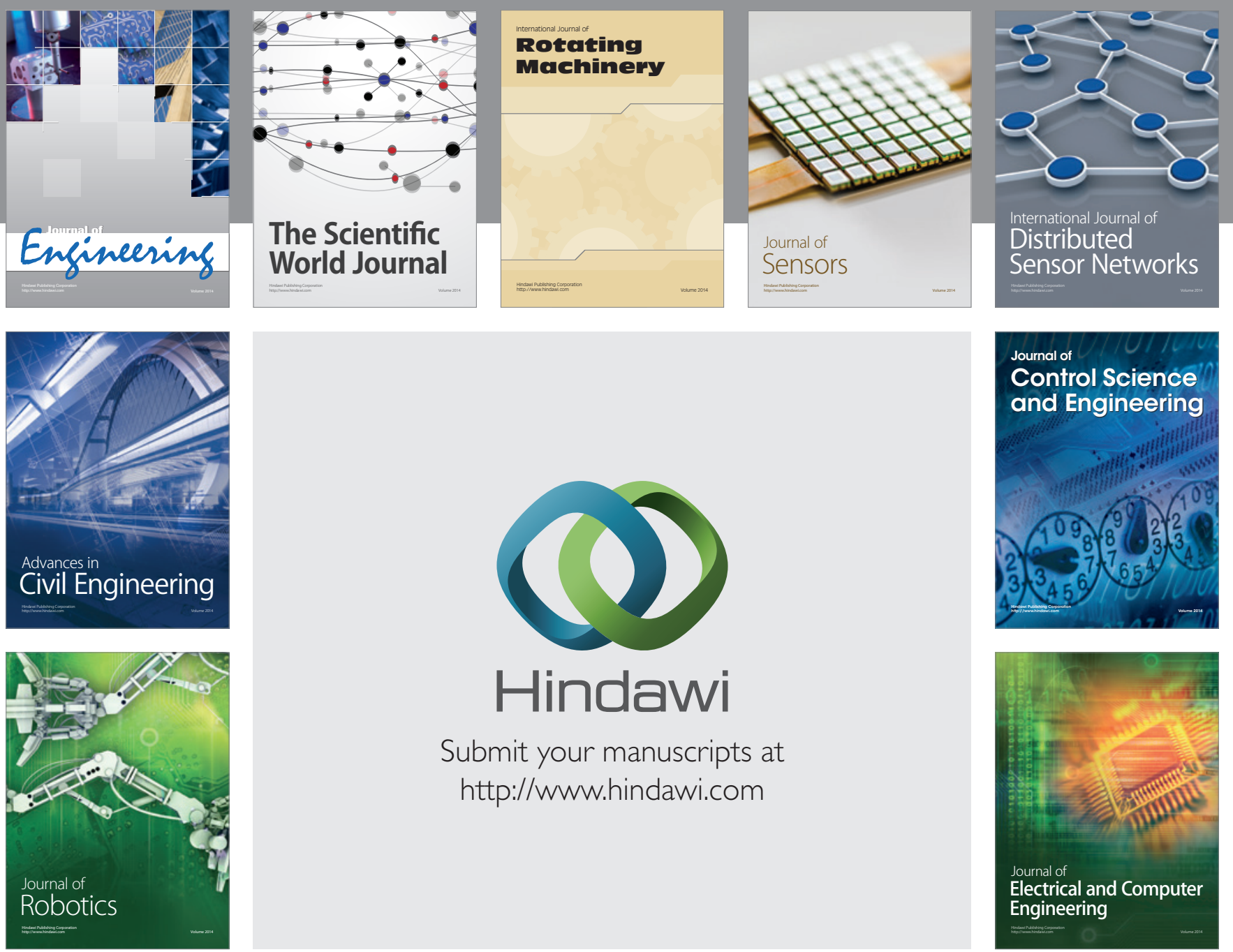

Submit your manuscripts at

http://www.hindawi.com
